\title{
Nonlinear dynamic analysis of an inclined Timoshenko beam subjected to a moving mass/force with beam's weight included
}

\author{
Ahmad Mamandi ${ }^{\mathrm{a}, *}$ and Mohammad H. Kargarnovin ${ }^{\mathrm{b}}$ \\ ${ }^{a}$ Mechanical and Aerospace Engineering Department, Science and Research Branch, Islamic Azad University, \\ Tehran, Iran \\ ${ }^{\mathrm{b}}$ Mechanical Engineering Department, Sharif University of Technology, Tehran, Iran
}

Received 4 September 2009

Revised 7 June 2010

\begin{abstract}
In this study, the nonlinear vibrations analysis of an inclined pinned-pinned self-weight Timoshenko beam made of linear, homogenous and isotropic material with a constant cross section and finite length subjected to a traveling mass/force with constant velocity is investigated. The nonlinear coupled partial differential equations of motion for the rotation of warped cross-section, longitudinal and transverse displacements are derived using the Hamilton's principle. These nonlinear coupled PDEs are solved by applying the Galerkin's method to obtain dynamic responses of the beam. The dynamic magnification factor and normalized time histories of mid-point of the beam are obtained for various load velocity ratios and the outcome results have been compared to the results with those obtained from linear solution. The influence of the large deflections caused by a stretching effect due to the beam's fixed ends is captured. It was seen that existence of quadratic-cubic nonlinear terms in the nonlinear governing coupled PDEs of motion causes stiffening (hardening) behavior of the dynamic responses of the self-weight beam under the act of a traveling mass as well as equivalent concentrated moving force. Furthermore, in a case where the object leaves the beam, its planar motion path is derived and the targeting accuracy is investigated and compared with those from the rigid solution assumption.
\end{abstract}

Keywords: Nonlinear vibrations, inclined self-weight Timoshenko beam, moving mass/force, Galerkin's method, projectile targeting

\section{Introduction}

The vibration analysis of structural elements, such as strings, rods, beams, plates and shells under the act of a traveling mass/force is of considerable practical importance and many analytical and numerical methods have been proposed in the past decades to investigate the linear and nonlinear dynamic behavior of such systems. Of great importance would be the study of dynamical behavior of composite and FGM made engineering materials from engineering point of view. However, until now almost no attention has been paid to the study of nonlinear dynamic analysis for the coupled warping bending rotation of the cross-section, longitudinal and transverse deflections of an inclined short and thick beam with self-weight subjected to a moving mass/force. It is believed that this study is the first attempt to initiate and investigate the topic under study.

* Corresponding author: Ahmad Mamandi, Ph.D. of Aerospace Engineering, Design of Aerospace Structures, Post Doctoral Research Fellow. E-mail: am_2001h@yahoo.com. 
It should be noted that the bridges on which vehicles or trains travel and trolleys of overhead traveling cranes that move on their girders may be modeled as moving loads on simply supported beams. Also, in aerospace industries the rocket launcher systems and fire arms are some applications related to vibrations of the inclined beams.

A comprehensive treatment of the subject of vibrations of structures due to moving loads which contains a large number of related cases is studied by Fryba [1]. In [2], an integral transform method to tackle with issues of moving force traveling on infinite length beams is studied. On the other hand in order to deal with problems including finite length beams, modal analysis is commonly used. A dynamic Green function approach is used to determine the response of a finite length simply supported Euler-Bernoulli beam subjected to a moving load in [3] and the authors proposed a simple matrix expression for the deflection of the beam. In [4], the effect of a moving mass on the dynamic behavior of a simply supported Euler-Bernoulli beam was studied. The linear finite element analysis was used to investigate dynamic behavior of a simply supported Euler-Bernoulli beam under the act of moving loads by [5-8]. The linear vibration analysis using the Galerkin's technique for a Timoshenko beam traversed by a moving mass was applied in [9] and this work was verified by [10] using the linear finite element method. The effect of weight of the beam on the dynamic behavior of a horizontal simply supported Timoshenko beam subjected to moving forces was studied in [11] using the Galerkin's approach. The linear dynamic analysis of a simply-supported Timoshenko beam under the act of a moving mass has been studied in [12]. In [13], the finite difference technique was used in the dynamic analysis of a simple beam traversed by uniform partially moving masses. The vibration analysis of multi-span Timoshenko beams and frames due to moving loads were studied by [14,15], respectively. In [16], the coupled longitudinal and transverse vibrations of a horizontal simply supported Euler-Bernoulli beam with beam's weight included traversed by a moving mass has been studied using Hamilton's principle to obtain two nonlinear coupled differential equations of motion. In that paper a finite difference method combined with a perturbation technique has been used to solve the resulting boundary value problem governing the transverse and longitudinal displacements of the beam. The dynamic responses of a simply supported Euler-Bernoulli beam subjected to a constant velocity moving force is discussed in [17] and both analytical and linear finite element solutions are presented. Nonlinear dynamical behavior of Timoshenko beams with infinite length under the act of traveling harmonic loads resting on Pasternak viscoelastic foundations are studied in [18,19]. In [20], dynamic responses of an inclined weightless Euler-Bernoulli beam subjected to a moving mass has been investigated using the linear finite element method considering transverse and longitudinal displacements for the beam. The nonlinear vibration of a horizontal pinned-pinned Euler-Bernoulli beam traversed by a moving mass considering the influence of the load inertia and the geometric nonlinearity caused by stretching effect of mid-plane of the beam due to the fixed supports was studied in [21]. In that analysis the effect of longitudinal inertia and longitudinal damping in the governing equations of motion has been neglected. Moreover, their approximate-analytical solution is based on the multiple time scales method (MTS) for the system having cubic nonlinearity. In [22], the nonlinear dynamic analysis of an eccentrically pre-stressed viscoelastic beam under the act of a concentrated moving harmonic force has been studied. In that paper, the nonlinear deflection behavior of the beam is approximated by some polynomial functions and material of the beam was assumed to follow the Kelvin-Voigt model. Furthermore, the effects of large deflections, the velocity of the moving harmonic load, the pre-stress load and the excitation frequency were discussed. Also, the hardening behavior of the beam was investigated in the obtained results. The transverse vibrations of a standing Timoshenko beam under influence of self-weight of the beam is studied by [23] using the multiple time scales method. The linear analysis for beams has been studied in [24] using Newmark- $\beta$ method to solve the discrete equations of motion for thin and thick beam theories neglecting the longitudinal displacement. The nonlinear dynamic analysis of an inclined Euler-Bernoulli beam with finite length subjected to a concentrated moving force has been studied in [25]. The nonlinear coupled PDEs of motion were solved by using mode summation method. The authors concluded that the quadratic nonlinearity assumption in the mode equations of motion prevail the softening behavior on the dynamic response of the beam. Moreover, the steady-state responses of the beam were studied by using the multiple time scales method. They have shown that both saturation phenomenon and jump phenomenon can be predicted in the internal-external primary resonance condition for the longitudinal excitation. Recently, nonlinear vibration analysis of a finite length Euler-Bernoulli beam traversed by a moving force resting on a nonlinear viscoelastic foundation has been studied in [26]. The Galerkin's method has been used to discretize the nonlinear PDE of motion governing lateral displacement of the beam. The authors also used the multiple time scale method to investigate internal-external resonance conditions of the beam. 


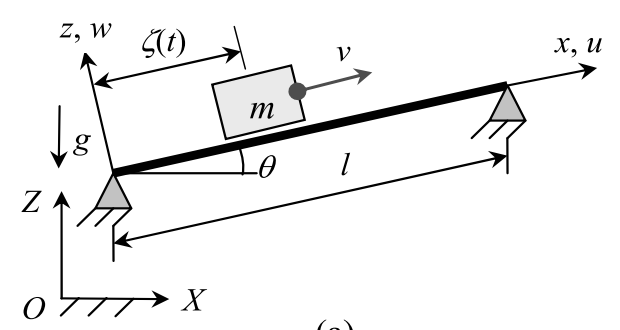

(a)

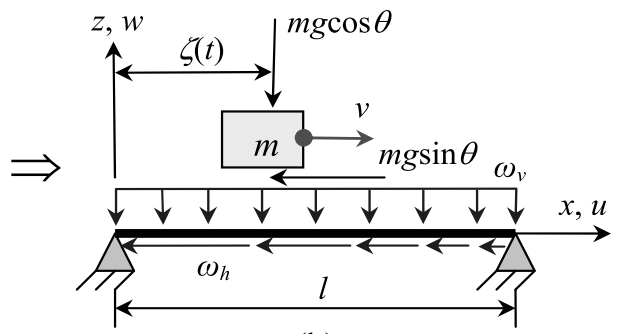

(b)

Fig. 1. (a) A mass traveling on an inclined pinned-pinned Timoshenko beam; (b) Equivalent moving mass model on a self-weight Timoshenko beam with fixed ends.

From experimental point of view, it appears that as the amplitude of oscillation increases, nonlinear effects come into play (see Ref. [26]); therefore by considering that the source of nonlinearity may be due to either of; inertial, geometric or material in nature, the influence of the such terms on the beam dynamic behavior should also be included (see Ref. [27]). In the present study, attention is put on geometric nonlinearity which may be caused by large curvatures and nonlinear stretching of the mid-plane of an inclined self-weight Timoshenko beam with fixed ends using von-Karman strain-displacement relations in the moderately large deflection theory. In general, due to existence of nonlinear terms, the exact analytical (closed form) solutions for equations of motions are not available.

This study is focused on the Galerkin's method to solve the nonlinear governing coupled PDEs of motion for the rotation of warped cross-section, transverse and longitudinal vibrations of an inclined self-weight Timoshenko beam under the act of a moving mass/force which is derived using Hamilton's principle. The modal equations can be solved numerically using the Adams-Bashforth-Moulton integration method via MATLAB solver package. The appropriate parametric study by taking into account the effects of the magnitude of the traveling mass or equivalent concentrated force, the velocity of the traveling mass/force, beam's inclination angle are carried out. It should be noted that in the present study the nonlinear effects of axial strain, bending curvature and shear strain on the dynamic responses of the beam are all considered by applying the von-Karman strain-displacement relations.

\section{Mathematical modeling}

An inclined Timoshenko beam with length of $l$ and inclination angle of $\theta$ traveled by a point mass of $m$ with constant velocity of $v$ is considered (see Fig. 1-a). The inclined beam in a gravity field is subjected to a linearly varying axial force and a constant magnitude lateral force due to the weight of the beam $[16,23,28]$. The longitudinal and lateral gravity-induced components of weight of the beam per unit length are $\rho A g \sin \theta(1-x / l)$ and $\rho A g \cos \theta$, respectively as shown by $\omega_{h}$ and $\omega_{v}$ in Fig. 1-b. Moreover, the longitudinal and lateral gravity-induced components of the force with which the traveling point mass acts on the beam are $m g \cos \theta$ and $m g \sin \theta$, respectively as shown in Fig. 1-b. To make derivation of the motion's governing equations, it is further assumed that the friction force between beam and the mass is negligible (please see Ref. [20]). In our up-coming analysis, it is to be mentioned that when the mass enters the left end of the beam, zero initial conditions for the beam are assumed, i.e. the beam is at rest at time $t=0$. It is further assumed that the mass will be in full contact with the beam during its motion, i.e. no separation occurs. The nonlinear dynamic behavior for the coupled bending rotation of warped cross-section, longitudinal and transversal displacements of a uniform inclined Timoshenko beam under the act of a moving mass and weight of the beam is considered. The beam supports are restricted from any axial movement and hence, this will produce mid-plane stretching which causes to create further tensile/compressive internal axial force. Furthermore, it is assumed that the dampings of any kinds are negligible. Moreover, the beam deforms within the linear elastic range and therefore Hooke's laws are prevailing. In this study the Mindlin plate theory [11] is employed and the von-Karman's strain-displacement relations are as:

$$
\varepsilon_{x x}=u_{, x}+\frac{1}{2} w_{, x}^{2}, \quad \gamma_{x z}=-\psi+w_{, x} \text { and } \kappa_{x}=w_{, x x}
$$


in which $u=u(x, t)$ is the axial longitudinal time dependent in-plane displacement, $w=w(x, t)$ is the time dependent transverse deflection of the beam measured upward from its equilibrium position when unloaded and $\psi=\psi(x, t)$ is the rotation of the warped cross-section of the beam due to the bending. It is further to be noted that the subscripts $(, t)$ and $(, x)$ stand for the derivative with respect to the time $(t)$ and spatial coordinate $(x)$ to the related order, respectively. In addition, $\varepsilon, \gamma$ and $\kappa$ are the longitudinal (normal) strain, shear strain and curvature at the center line of the Timoshenko beam, respectively. To obtain the governing differential equations of motion by applying the Hamilton's principle the kinetic energy, $K$, and the strain energy, $U$, of the beam are [28]:

$$
\begin{aligned}
& K=\frac{1}{2} \int_{0}^{l} \rho\left(A u_{, t}^{2}+A w_{, t}^{2}+I \psi_{, t}^{2}\right) d x \\
& U=\frac{1}{2} \int_{0}^{l}\left(E A \varepsilon_{x x}^{2}+E I \kappa_{x}^{2}+k G A \gamma_{x z}^{2}-\rho A g \sin \theta(l-x) w_{, x}^{2}\right) d x
\end{aligned}
$$

also the total work done by the inertial force due to the action of the traveling mass and the gravity field is $[16,20$, 28]:

$$
W_{e}=-\int_{0}^{l}\left(m\left(g \sin \theta+u_{, t t}\right) u+m\left(g \cos \theta+w_{, t t}+2 v w_{, x t}+v^{2} w_{, x x}\right) w+\rho A g \cos \theta w\right) d x
$$

in which $m w_{, t t}, m u, t t, 2 m v w_{, x t}$ and $m v^{2} w_{, x x}$ represent different inertial forces, Coriolis force and centrifugal force acting on the beam respectively due to the fact that the motion of the traveling mass is on the deformed shape of the beam. Now, we can establish the Lagrangian function of the system as: $L=K-\left(U-W_{e}\right)$. Applying the Hamilton's principle on $L$ as: $\delta \int_{t_{1}}^{t_{2}} L d t=0$ or:

$$
\delta \int_{t_{1}}^{t_{2}}(U-K) d t=\int_{t_{1}}^{t_{2}} \delta W_{e} d t
$$

by doing some mathematics, one would get the nonlinear governing coupled PDEs of the motion (EOMs) for the problem under consideration as follows:

Moment relation in $\psi$ direction:

$$
E I \psi_{, x x}+k A G\left(w_{, x}-\psi\right)-\rho I \psi_{, t t}=0
$$

Force relation in $z$ direction:

$$
\begin{aligned}
\rho A w_{, t t}+k A G\left(\psi_{, x}-w_{, x x}\right)-E A\left(u_{, x x} w_{, x}+u_{, x} w_{, x x}+\frac{3}{2} w_{, x x} w_{, x}^{2}\right)+\rho A g \sin \theta\left[(l-x) w_{, x x}-w_{, x}\right] \\
=-\rho A g \cos \theta-m \delta(x-\zeta) \chi(t)\left(w_{, t t}+2 v w_{, x t}+v^{2} w_{, x x}+g \cos \theta\right)_{x=\zeta}
\end{aligned}
$$

Force relation in $x$ direction:

$$
\rho A u_{, t t}-E A\left(u_{, x x}+w_{, x} w_{, x x}\right)=-m \delta(x-\zeta) \chi(t)\left(u_{, t t}+g \sin \theta\right)_{x=\zeta}
$$

where, $\rho$ is the beam density, $A$ is the cross-sectional area of the beam, $I$ is the beam's cross-sectional second moment of inertia, $E$ is Young's modulus, $G$ is shear modulus, $k$ is the shear correction factor, $E I$ is the beam's flexural rigidity, $\rho A$ is the beam's mass per unit length, $m$ is the traveling mass and $g$ is the gravitational acceleration. Furthermore, $\delta(x-\zeta)$ is the Dirac's delta function in which $\zeta$ is the instantaneous position of the moving mass with the velocity of $v$ on the beam such that: $\zeta=v t$. Moreover, $\chi(t)$ is the pulse function which is equal to one while the mass is traveling on the beam and is equal to zero, when the traveling mass is outside the beam span, that is: $\chi(t)=u(t)-u(t-l / v)$, in which $u(t)$ represents the unit step function.

\section{Method of solution}

In this study the conventional series expansion solution based on modal analysis approach also called the Galerkin's method is considered to analyze the vibrations of an inclined self-weight Timoshenko beam with pinned-pinned ends 
under the act of a moving mass/force. Based on the separation of variables technique, the response of Timoshenko beam in terms of the linear free-oscillation modes can be assumed as follows [28]:

$$
\begin{aligned}
& w(x, t)=\sum_{j=1}^{n} \phi_{j}(x) p_{j}(t)=\Phi^{T}(x) \mathbf{P}(t) \\
& \psi(x, t)=\sum_{j=1}^{n} \psi_{j}(x) q_{j}(t)=\Psi^{T}(x) \mathbf{Q}(t) \\
& u(x, t)=\sum_{j=1}^{n} \theta_{j}(x) r_{j}(t)=\Theta^{T}(x) \mathbf{R}(t)
\end{aligned}
$$

where $\mathbf{P}(t), \mathbf{Q}(t)$ and $\mathbf{R}(t)$ are vectors of order $n$ listing the generalized coordinate $p_{j}(t), q_{j}(t)$ and $r_{j}(t)$, respectively and $\Phi(x), \Psi(x)$ and $\Theta(x)$ are some vector functions collecting the first $n$ eigen-functions (mode shapes) of $\phi_{j}(x)$, $\psi_{j}(x)$ and $\theta_{j}(x)$, respectively.

By substituting Eqs (8), (9) and (10) into Eqs (5), (6) and (7), pre-multiplying both sides of Eqs (5), (6) and (7) by $\Psi^{T}(x), \Phi^{T}(x)$ and $\Theta^{T}(x)$, respectively, integrating over the interval $(0, l)$ and imposing the properties of the Dirac's delta function, the resulting nonlinear coupled ODEs of motion in matrix form can be expressed as follows:

$$
\begin{aligned}
& -\rho I \sum_{j=1}^{n} \mathbf{S}_{i j} \ddot{q}_{j}(\mathrm{t})+\sum_{j=1}^{n}\left[E I \mathbf{K}_{i j}-k A G \mathbf{S}_{i j}\right] q_{j}(\mathrm{t})+k A G \sum_{j=1}^{n} \mathbf{E}_{i j} p_{j}(t)=0, \quad i=1,2, \ldots, n \\
& \sum_{j=1}^{n}\left[\rho A \mathbf{M}_{i j}+m \mathbf{B}_{i j}(t) \chi(t)\right] \ddot{p}_{j}(t)+2 m v \chi(t) \sum_{j=1}^{n} \mathbf{A}_{i j}(t) \dot{p}_{j}(t)+ \\
& \sum_{j=1}^{n}\left[m v^{2} \mathbf{C}_{i j}(t) \chi(t)-k A G \mathbf{H}_{i j}+\rho A g \sin \theta\left(-\mathbf{N} \mathbf{1}_{i j}+\mathbf{N} \mathbf{2}_{i j}\right)\right] p_{j}(t)+k A G \sum_{j=1}^{n} \mathbf{F}_{i j} q_{j}(t) \\
& -E A \sum_{j=1}^{n} \sum_{k=1}^{n} r_{j}(t) \mathbf{G}_{i j k} p_{k}(t)-E A \sum_{j=1}^{n} \sum_{k=1}^{n} p_{j}(t) \mathbf{T}_{i j k} r_{k}(t)-\frac{3}{2} E A \sum_{j=1}^{n} \sum_{k=1}^{n} p_{j}(t) \mathbf{I}_{i j k} p_{k}(t)^{2} \\
& =-\rho A g \cos \theta \mathbf{N} \mathbf{3}_{i}-m g \cos \theta \chi(t) \mathbf{b}_{i}(t), \quad i=1,2, \ldots, n
\end{aligned}
$$

and

$$
\begin{aligned}
& \sum_{j=1}^{n}\left[\rho A \mathbf{J}_{i j}+m \mathbf{O}_{i j}(t) \chi(t)\right] \ddot{r}_{j}(t)-E A \sum_{j=1}^{n} \mathbf{N}_{i j} r_{j}(t)-E A \sum_{j=1}^{n} \sum_{k=1}^{n} p_{j}(t) \mathbf{L}_{i j k} p_{k}(t) \\
& =-m g \sin \theta \chi(t) \mathbf{d}_{i}(t), \quad i=1,2, \ldots, n
\end{aligned}
$$

in which the matrices $\mathbf{S}, \mathbf{K}, \mathbf{L}, \mathbf{M}, \mathbf{N}, \mathbf{N 1}, \mathbf{N 2}, \mathbf{N 3}, \mathbf{A}, \mathbf{B}, \mathbf{C}, \mathbf{O}, \mathbf{I}, \mathbf{J}, \mathbf{T}, \mathbf{E}, \mathbf{F}, \mathbf{G}$ and $\mathbf{H}$ are defined as:

$$
\begin{aligned}
& (\mathbf{M})_{i j}=\int_{0}^{l} \phi_{i}(x) \phi_{j}(x) d x, \quad(\mathbf{H})_{i j}=\int_{0}^{l} \phi_{i}(x) \phi_{j}^{\prime \prime}(x) d x, \quad(\mathbf{F})_{i j}=\int_{0}^{l} \phi_{i}(x) \psi_{j}^{\prime}(x) d x, \\
& (\mathbf{S})_{i j}=\int_{0}^{l} \psi_{i}(x) \psi_{j}(x) d x, \quad(\mathbf{K})_{i j}=\int_{0}^{l} \psi_{i}(x) \psi_{j}^{\prime \prime}(x) d x, \quad(\mathbf{E})_{i j}=\int_{0}^{l} \psi_{i}(x) \phi_{j}^{\prime}(x) d x, \quad(\mathbf{N})_{i j}=\int_{0}^{l} \theta_{i}(x) \theta_{j}^{\prime \prime}(x) d x, \quad(\mathbf{N} \mathbf{1})_{i j}=\int_{0}^{l} \phi_{i}(x) \phi_{j}^{\prime}(x) d x, \\
& (\mathbf{J})_{i j}=\int_{0}^{l} \theta_{i}(x) \theta_{j}(x) d x, \quad(\mathbf{N} \mathbf{3})_{i}=\int_{0}^{l} \phi_{i}(x) d x,
\end{aligned}
$$




$$
\begin{array}{ll}
(\mathbf{G})_{i j k}=\int_{0}^{l} \phi_{i}(x) \theta_{j}^{\prime \prime}(x) \phi_{k}^{\prime}(x) d x, & (\mathbf{I})_{i j k}=\int_{0}^{l} \phi_{i}(x) \phi_{j}^{\prime \prime}(x) \phi_{k}^{\prime 2}(x) d x, \\
(\mathbf{L})_{i j k}=\int_{0}^{l} \theta_{i}(x) \phi_{j}^{\prime \prime}(x) \phi_{k}^{\prime}(x) d x, & (\mathbf{T})_{i j k}=\int_{0}^{l} \phi_{i}(x) \phi_{j}^{\prime \prime}(x) \theta_{k}^{\prime}(x) d x, \\
(\mathbf{A})_{i j}=\phi_{i}(x=\zeta) \phi_{j}^{\prime}(x=\zeta), & (\mathbf{B})_{i j}=\phi_{i}(x=\zeta) \phi_{j}(x=\zeta), \\
(\mathbf{C})_{i j}=\phi_{i}(x=\zeta) \phi_{j}^{\prime \prime}(x=\zeta), & (\mathbf{O})_{i j}=\theta_{i}(x=\zeta) \theta_{j}(x=\zeta),
\end{array}
$$

where $i, j, k=1,2,3, \ldots, n$.

where prime and dot marks over any parameter indicates the derivative with respect to the position $(x)$ and time $(t)$, respectively. Furthermore, the $n \times 1$ column vectors of $\mathbf{b}$ and $\mathbf{d}$ are defined as:

$$
(\mathbf{b})_{i}=\phi_{i}(x=\zeta) \text { and }(\mathbf{d})_{i}=\theta_{i}(x=\zeta)
$$

It is clear that Eqs (11), (12) and (13) are three nonlinear coupled second order ordinary differential equations (ODEs). Also the boundary conditions for a pinned-pinned Timoshenko beam with fixed ends are as:

Essential BC's: $u(0, t)=u(l, t)=0 \quad$ therefore: $\theta=0 \quad$ at $\quad x=0$ and $l$

$$
w(0, t)=w(l, t)=0 \quad \text { therefore: } \quad \phi=0 \quad \text { at } \quad x=0 \text { and } l
$$

Natural BC: $M(0, t)=M(l, t)=0 \quad$ therefore: $\quad \psi_{, x}=0 \quad$ at $\quad x=0$ and $l$

and initial conditions are:

$$
\text { IC's: } u(x, 0)=u_{, t}(x, 0)=w(x, 0)=w_{, t}(x, 0)=\psi(x, 0)=\psi_{, t}(x, 0)=0
$$

The equations of motion for a Timoshenko beam with self-weight subjected to a moving concentrated force of magnitude $m g$ can be derived from the equations of motion for a Timoshenko beam with self-weight subjected to a moving mass by neglecting the inertial effect of the traveling mass. These resulting nonlinear coupled ODEs of motions are given by:

$$
\begin{gathered}
-\rho I \sum_{j=1}^{n} \mathbf{S}_{i j} \ddot{q}_{j}(\mathrm{t})+\sum_{j=1}^{n}\left[E I \mathbf{K}_{i j}-k A G \mathbf{S}_{i j}\right] q_{j}(\mathrm{t})+k A G \sum_{j=1}^{n} \mathbf{E}_{i j} p_{j}(t)=0, \quad i=1,2, \ldots, n \\
\rho A \sum_{j=1}^{n} \mathbf{M}_{i j} \ddot{p}_{j}(t)+\sum_{j=1}^{n}\left[-k A G \mathbf{H}_{i j}+\rho A g \sin \theta\left(-\mathbf{N} \mathbf{1}_{i j}+\mathbf{N} \mathbf{2}_{i j}\right)\right] p_{j}(t) \\
+k A G \sum_{j=1}^{n} \mathbf{F}_{i j} q_{j}(t)-E A \sum_{j=1}^{n} \sum_{k=1}^{n} r_{j}(t) \mathbf{G}_{i j k} p_{k}(t)-E A \sum_{j=1}^{n} \sum_{k=1}^{n} p_{j}(t) \mathbf{T}_{i j k} r_{k}(t) \\
-\frac{3}{2} E A \sum_{j=1}^{n} \sum_{k=1}^{n} p_{j}(t) \mathbf{I}_{i j k} p_{k}(t)^{2}=-\rho A g \cos \theta \mathbf{N} \mathbf{3}_{i}-m g \cos \theta \chi(\mathrm{t}) \mathbf{b}_{i}(t), \\
i=1,2, \ldots, n
\end{gathered}
$$

and

$$
\begin{gathered}
\rho A \sum_{j=1}^{n} \mathbf{J}_{i j} \ddot{r}_{j}(t)-E A \sum_{j=1}^{n} \mathbf{N}_{i j} r_{j}(t)-E A \sum_{j=1}^{n} \sum_{k=1}^{n} p_{j}(t) \mathbf{L}_{i j k} p_{k}(t)=-m g \sin \theta \chi(\mathrm{t}) \mathbf{d}_{i}(t), \\
i=1,2, \ldots, n
\end{gathered}
$$

In order to solve the Eqs (11), (12) and (13), all entries in the matrices in Eqs (14) and (15) should be calculated. Herein, by inspection it can be seen that the following functions for $\phi(x), \psi(x)$ and $\theta(x)$ will satisfy the boundary conditions $[9,28]$ :

$$
\phi_{n}(x)=\sin \left(\frac{n \pi x}{l}\right), \quad \psi_{n}(x)=\cos \left(\frac{n \pi x}{l}\right) \text { and } \theta_{n}(x)=\sin \left(\frac{n \pi x}{l}\right), \text { with } n=1,2,3, \ldots
$$


By inserting the above functions into the Eqs (14) and (15) all entries in all matrices can be calculated. In the next step these evaluated matrices will be inserted in the Eqs (11), (12) and (13) and the later set of equations can be solved numerically using the Adams-Bashforth-Moulton integration method via MATLAB solver package out of which the values of $p_{n}(t), q_{n}(t)$ and $r_{n}(t)$ can be obtained. By back substitution of $p_{n}(t), q_{n}(t)$ and $r_{n}(t)$ in Eqs (9), (10) and (11), $u(x, t), w(x, t)$ and $\psi(x, t)$ are established, respectively. In the next step based on the obtained values for $u(x, t)$, $w(x, t)$ and $\psi(x, t)$ the dynamic response of the beam under variation of different parameters namely; velocity of the concentrated moving mass or an equivalent force, beam's inclination angle and magnitude of the moving load are studied. Finally as an application of this study the targeting accuracy of planar motion of a projectile when leaves an inclined beam is presented.

\section{Verifications and case studies}

It has to be mentioned that the obtained results for the problem under study are based on the summation of first ten modes of assumed excited eigen-functions i.e. $n=1$ to 10 . The reason for adopting the first ten modes is based on a separate convergence study on $u(x, t), w(x, t)$ and $\psi(x, t)$ which indicated that for $n<10$ the obtained results will not be satisfactory $[9,11]$.

\subsection{Verification of results}

As it was mentioned in introduction section, at the moment no specific results exist for the problem under consideration in the literature. Therefore, to verify the validity of the obtained results in this study we have to consider some special cases of our study to be compared with those existing for weightless horizontal Timoshenko beams in the literature.

In the first attempt we set the higher order terms in Eqs (6) and (7) i.e. second parenthesis and the first parenthesis of their left hand sides, respectively equal to zero. This led us to a set of relations for $u(x, t), w(x, t)$ and $\psi(x, t)$ referred to the case known for linear analysis for weightless horizontal Timoshenko beams. To establish verifications of our analysis, we consider the data given in [12] as: $l=4.352 \mathrm{~m}, E=2.02 \times 10^{11} \mathrm{~N} / \mathrm{m}^{2}, G=7.7 \times 10^{10} \mathrm{~N} / \mathrm{m}^{2}$, $I=5.71 \times 10^{-7} \mathrm{~m}^{4}, k=0.7, \rho=15267 \mathrm{~kg} / \mathrm{m}^{3}, A=1.31 \times 10^{-3} \mathrm{~m}^{2}, m=21.8 \mathrm{~kg}, v=27.49 \mathrm{~m} / \mathrm{s}$ and $g=$ $9.806 \mathrm{~m} / \mathrm{s}^{2}$. Based on input above data and developed computer program for the linear analysis, we calculated the maximum deflection of the mid-point of the considered weightless beam as; $5.91 \mathrm{~mm}$, whereas the similar result reported by [29] and [12] are $5.84 \mathrm{~mm}$ and $5.78 \mathrm{~mm}$, respectively which show very good agreement between our numerical result and other references. However, as described before, in this study we focus on the nonlinear analysis of coupled longitudinal, transversal and cross-section rotational vibrations of an inclined self-weight beam under the act of a traveling mass.

To extend checking on the validity of obtained results, we consider a horizontal Timoshenko beam with the following parameters and material properties [9]: $l=1 \mathrm{~m}, E=207 \times 10^{9} \mathrm{~N} / \mathrm{m}^{2}, G=77.6 \times 10^{9} \mathrm{~N} / \mathrm{m}^{2}, k=0.9$, $\rho=7700 \mathrm{~kg} / \mathrm{m}^{3}, I=6.236 \times 10^{-5} \mathrm{~m}^{4}, A=0.02736 \mathrm{~m}^{2}, m=0.2 \rho A l \mathrm{~kg}, \alpha=0.11, g=9.81 \mathrm{~m} / \mathrm{s}^{2}$ and $\beta=0.15$, in which $\beta=\pi r_{0} / l$ is Rayleigh's slenderness coefficient with $r_{0}$ taken as the radius of gyration of the beam. Based on above data, the computer code was run for the linear case and the vertical displacement $(w)$ of the instantaneous positions of the moving mass are calculated and the dimensionless outcome results are depicted and compared with other existing results in Fig. 2. The normalization factor for the vertical displacement is $w_{s t}=m g l^{3} / 48 E I$ which is a mid-point deflection of a simply supported weightless beam under mid-span concentrated load of size $m g$. A close inspection of the curves in the Fig. 2 indicates good agreements between two results.

\subsection{Results and discussions}

In all following studied cases, the beam specifications are listed in Table 1, unless otherwise specified.

In order to have a better insight on interpreting the variation of the obtained results are presented in dimensionless forms. We begin by defining the dynamic magnification factor $D_{d}$ as the ratio of the maximum magnitude of the dynamic deflection at the mid-span of the beam to the maximum static deflection at the same location for a horizontal 
Table 1

Geometry and material properties of considered inclined beam

\begin{tabular}{lcclcc}
\hline Parameter & Symbol & Value & Parameter & Symbol & Value \\
\hline beam's cross-sectional area $\left(\mathrm{m}^{2}\right)$ & $A(b \times h)$ & $5 \times 10^{-3}(0.05 \times 0.1)$ & beam density $\left(\mathrm{kg} / \mathrm{m}^{3}\right)[9]$ & $\rho$ & 7850 \\
beam length $(\mathrm{m})$ & $l$ & 6 & Poisson coefficient [9] & $\nu$ & 0.25 \\
Young's modulus $\left(\mathrm{N} / \mathrm{m}^{2}\right)[9]$ & $E$ & $207 \times 10^{9}$ & shear correction factor [9,28] & $k$ & 0.85 \\
shear modulus $\left(\mathrm{N} / \mathrm{m}^{2}\right)[9]$ & $G$ & $77.6 \times 10^{9}$ & gravitational acceleration $\left(\mathrm{m} / \mathrm{s}^{2}\right)$ & $g$ & 9.81 \\
\hline
\end{tabular}

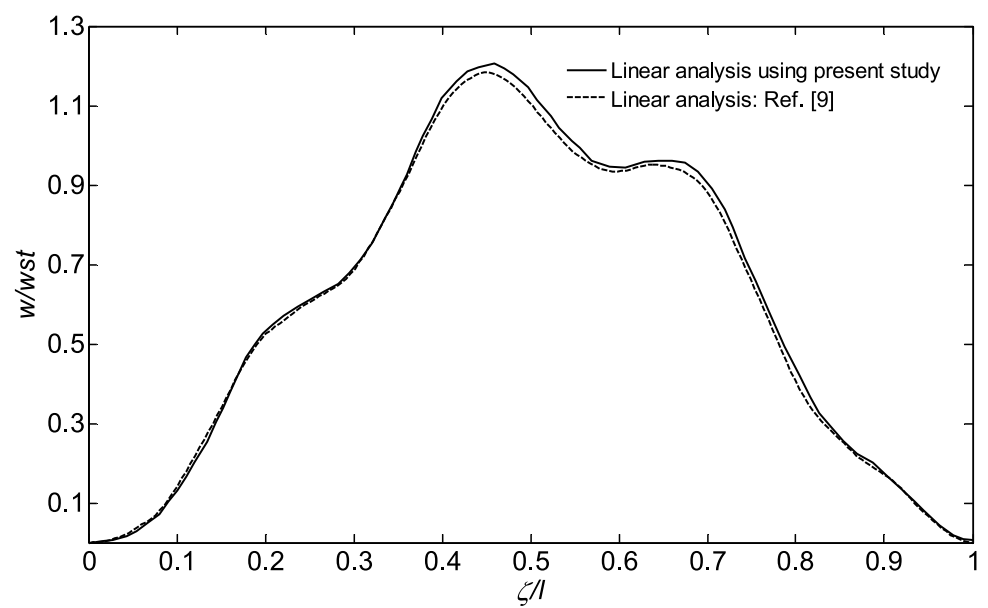

Fig. 2. Instantaneous normalized vertical displacement under a moving mass of $m$. (-) linear analysis using present study, (----) linear analysis: Ref. [9].

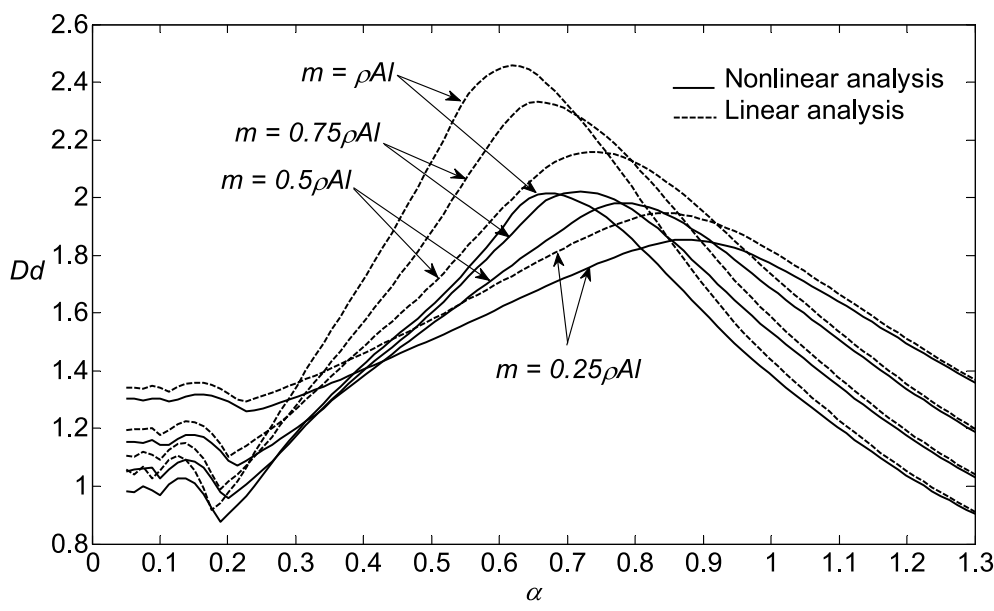

Fig. 3. Variation of dynamic magnification factor $\left(D_{d}\right)$ vs. $\alpha$ for an inclined self-weight Timoshenko beam with $\theta=36^{\circ}$ affected by a moving mass of $m(\mathrm{~kg})$. (-) nonlinear analysis, (--) linear analysis.

beam which is expressed by $w_{s t}=m g l^{3} / 48 E I+5 \rho g A l^{4} / 384 E I$. Also let's define the velocity ratio as $\alpha=v / v_{c r}$. It should be pointed out that $v_{c r}$ is the critical velocity of a concentrated moving force on a simply supported Euler-Bernoulli beam, i.e. $v_{c r}=(\pi / l) \sqrt{E I / \rho A}$ [9].

Based on the given data the analysis for the $D_{d}$ was conducted for both linear and nonlinear cases and its variation vs. $\alpha$ for different values of the moving mass ratios while the mass has not left the beam is depicted in the Fig. 3 . It can be seen that by increasing the magnitude of the moving mass the dynamic displacement response of the beam grows in such a way that does not follow the well-known linear trend. It can be observed that for $m=0.25 \rho A l$, the value of $D_{d}$ of the linear and nonlinear solutions have the lowest differences and for example the maximum $D_{d}$ 


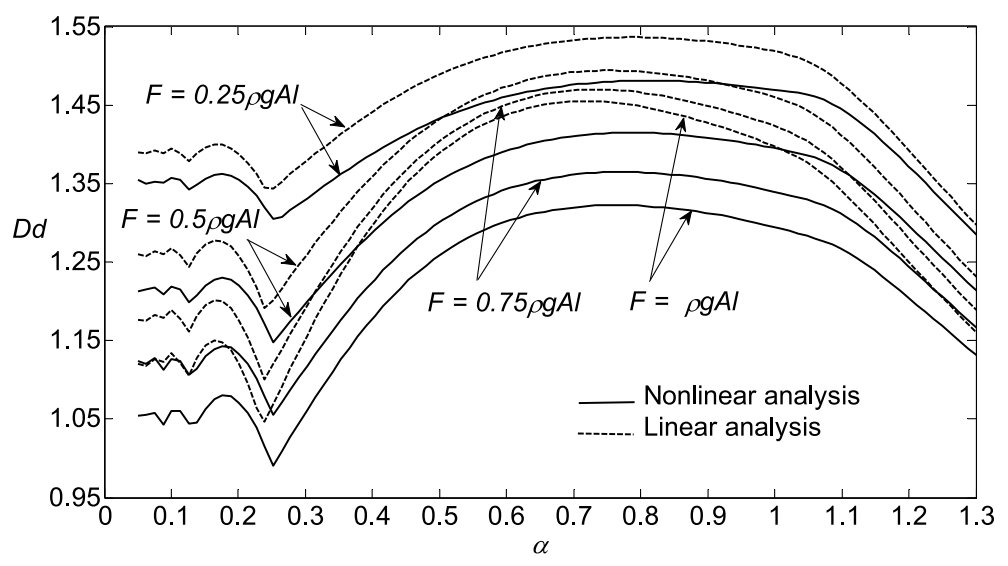

Fig. 4. Variation of dynamic magnification factor $\left(D_{d}\right)$ vs. $\alpha$ for an inclined self-weight Timoshenko beam with $\theta=36^{\circ}$ under motion of a concentrated moving force of $F(\mathrm{~N})$. ( $(-)$ nonlinear analysis, $(-)$ linear analysis.

for linear and nonlinear solutions yields 1.95 and 1.85 at $\alpha=0.84$ and $\alpha=0.87$, respectively. Moreover, as the value of $m$ increases, i.e. $m>0.25 \rho A l$ the difference between the linear and nonlinear dynamic response become more pronounced whereas for $m=\rho A l$ at $\alpha=0.62$ this difference has its maximum value of 0.45 which has about $22.5 \%$ increasing to the nonlinear case. In addition, another interesting observation on this figure reveals that for all values of $m$ and $\alpha$, the linear solution predicts higher value for $D_{d}$. The authors believe that the reason for this trend could be due to existence of strong coupling of longitudinal extension and transverse deflection of the beam which introduces more stiffness in the structure, while this effect can be picked up when one applies the nonlinear analysis. Meanwhile, in the region $0<\alpha<0.2$ another maxima is seen around $\alpha=0.13$ and similar differences between linear and nonlinear analyses as described above exists in this region. Briefly, in the under critical region $(\alpha \leqslant 0.9)$ the dynamic deflection of the beam generally increases by increasing the velocity of the traveling mass and in the overcritical region $(\alpha>0.9)$, the dynamic deflection decreases by increasing the velocity of the traveling mass. Furthermore, as a significant phenomenon it can be observed that by increasing the magnitude of the moving mass ratios the maximum $D_{d}$ for the linear and nonlinear solutions occurs in the lower values of $\alpha$.

By employing Eqs (18) to (20) one can obtain the variation of the dynamic magnification factor $D_{d}$ vs. $\alpha$ by changing the equivalent concentrated moving force. Figure 4 represents such variations for the mid-point of an inclined self-weight Timoshenko beam with inclination angle of $\theta=36^{\circ}$ under the act of several equivalent moving forces using the nonlinear as well as the linear analysis. It can be observed that the dynamic displacement response of the linear and nonlinear solutions have the lowest differences for $F=0.25 \rho g A l$ no matter what the value of $\alpha$ would be. For the same value of $F$, the maximum $D_{d}$ for linear and nonlinear solutions equals to 1.54 and 1.48 at $\alpha=0.8$ and $\alpha=0.9$, respectively. Moreover, as the value of $F$ increases, i.e. $F>0.25 \rho g A l$ the difference between the linear and nonlinear dynamic response become more distinct whereas for $F=\rho g A l$ at $\alpha \approx 0.72$ this difference has its maximum value of 0.14 which has about $9 \%$ reduction to the linear case. In addition, it can be seen from this figure that for all values of $F$ and $\alpha$, the linear solution predicts higher value for $D_{d}$. As it can be seen from Fig. 4, it should be noted that the linear equivalent moving force analysis does not predict always a unique value for the $D_{d}$ no matter what the value of $F$ would be.

In Fig. 5, the values of maximum dynamic response $(w)$ variation of a point at the middle of an inclined self-weight Timoshenko beam with inclination angle of $\theta=36 .^{\circ}$ vs. different values of the moving mass is shown for various velocity ratios of $\alpha=0.25,0.5,0.75,1$ and 1.25 , respectively using both linear and nonlinear solutions. It can be seen that the dynamic deflection of the nonlinear analysis is always lower than the one obtained from the linear solution. This incident which is known as the hardening behavior is mostly due to the existence of the coupled quadratic-cubic nonlinearity characteristic in the equations of motion of the beam, where in the other literature it is known as the equivalent to a nonlinear hard spring [27]. Also, it is seen from this figure that the dynamic mid-point displacements of such beam using linear and nonlinear solutions are almost the same for the value of $m \leqslant 0.2 \rho A l$. However, after this point, the magnitude of the $w$ of the nonlinear and the linear solutions differs gradually and the difference grows rapidly as the value of $m$ increases. Also, it can be observed from Fig. 5 that the difference 


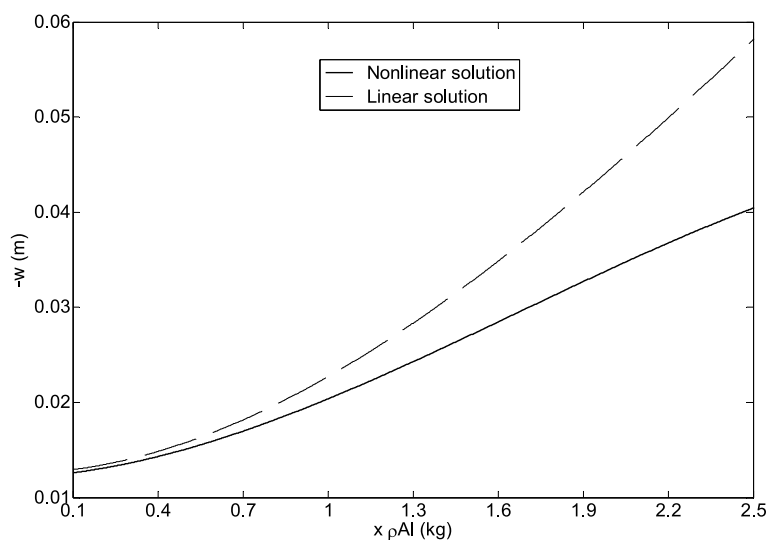

(a)

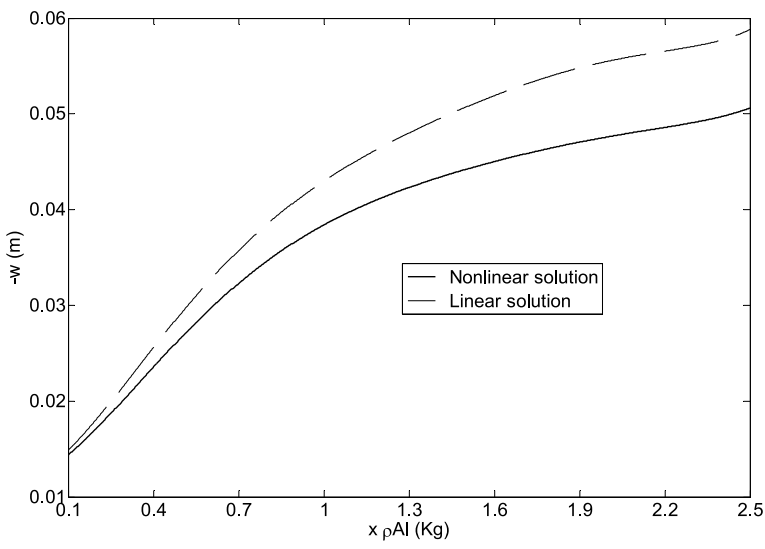

(c)

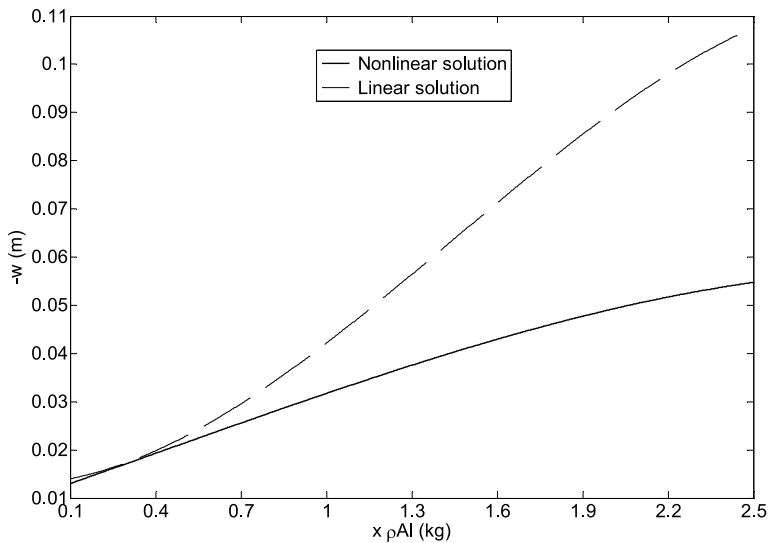

(b)

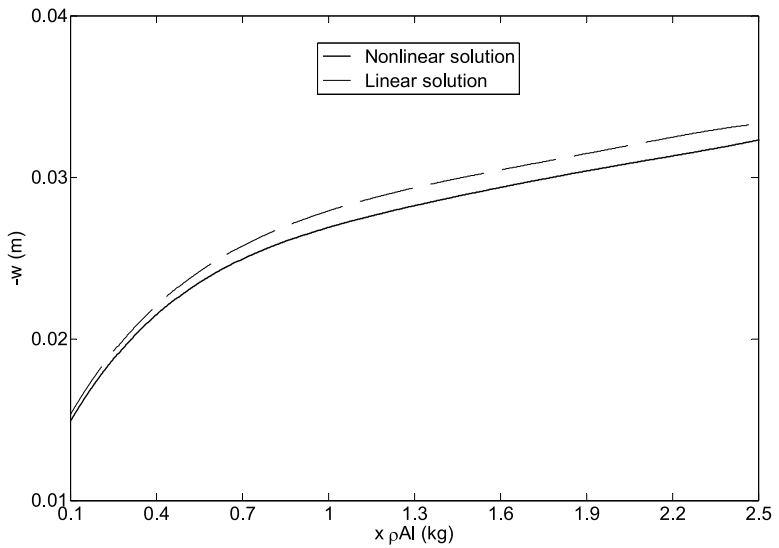

(d)

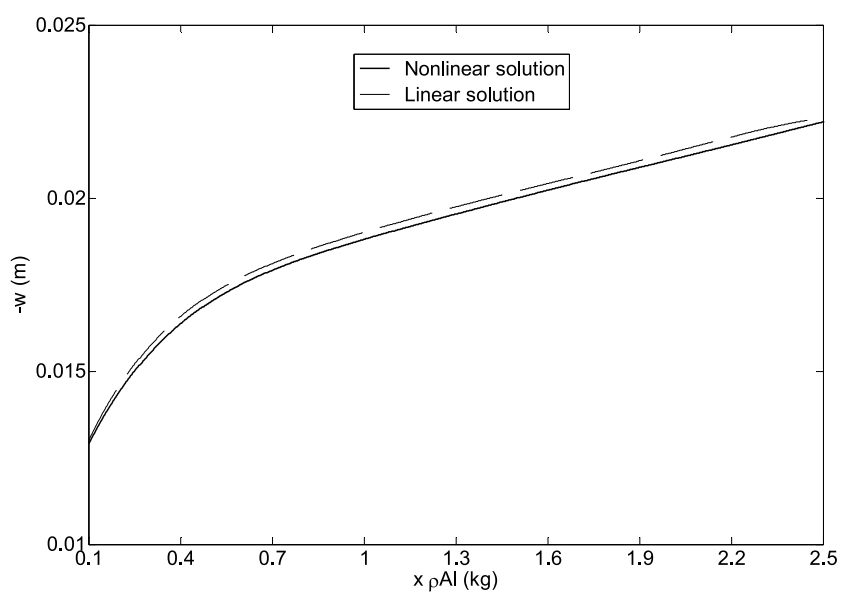

(e)

Fig. 5. Variation of the maximum dynamic response of a point at the middle of a self-weight Timoshenko beam with $\theta=36^{\circ}$ vs. different values of the moving mass for different velocity ratios; (a) $\alpha=0.25$, (b) $\alpha=0.5$, (c) $\alpha=0.75$, (d) $\alpha=1$, (e) $\alpha=1.25$. (—) nonlinear solution, (一) linear solution.

between the linear and the nonlinear solutions has an increasing trend up to the load velocity ratio of $\alpha=0.5$ and a reverse trend afterwards. It should be noted that the maximum difference between linear and nonlinear solutions for all cases in this figure occurs at $m=2.5 \rho A l$ at $\alpha=0.5$ (see Fig. 5-b). 


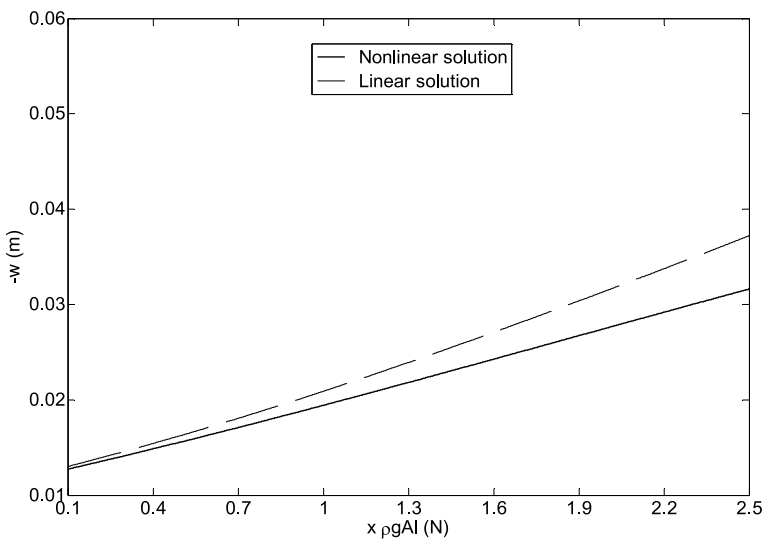

(a)

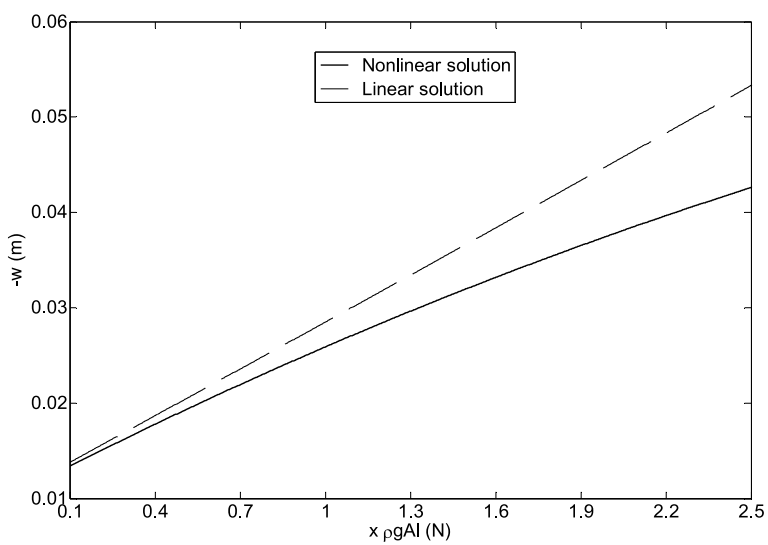

(c)

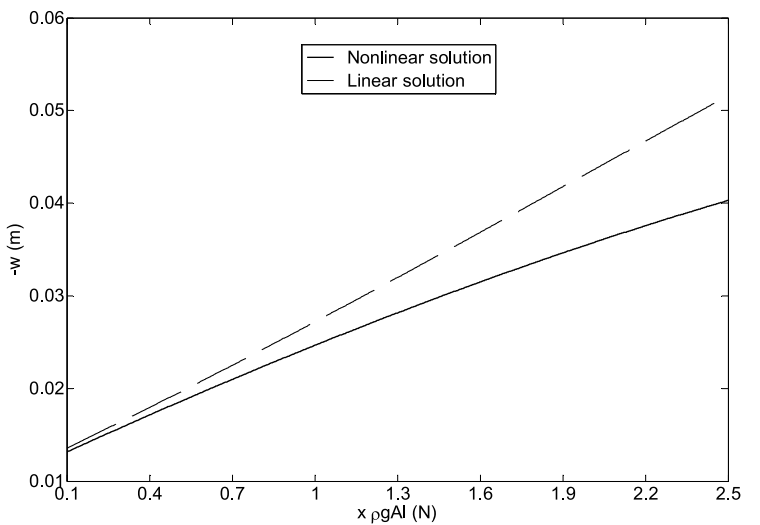

(b)

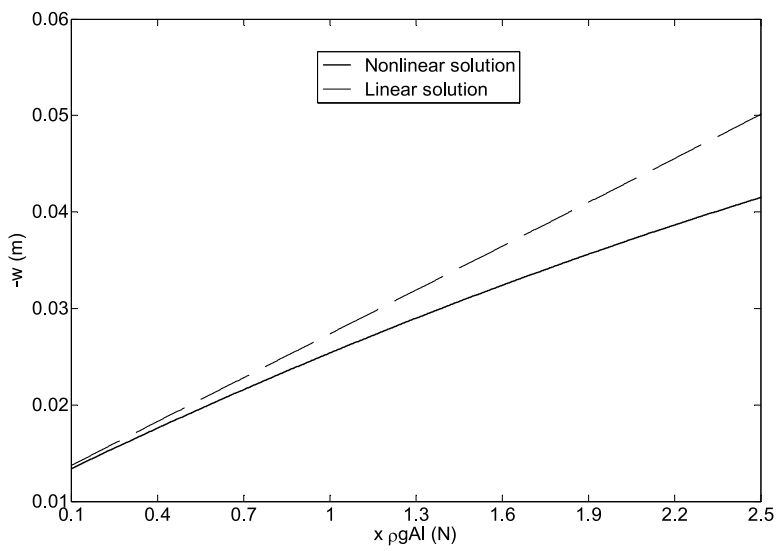

(d)

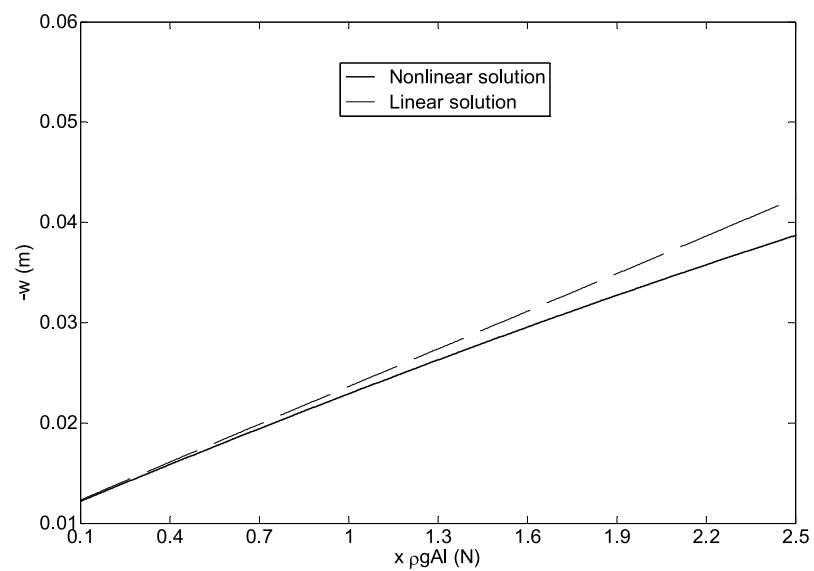

(e)

Fig. 6. Variation of the maximum dynamic response of a point at the middle of a self-weight Timoshenko beam with $\theta=36^{\circ}$ vs. different values of equivalent concentrated moving forces for different velocity ratios; (a) $\alpha=0.25$, (b) $\alpha=0.5$, (c) $\alpha=0.75$, (d) $\alpha=1$, (e) $\alpha=1.25$. ( - ) nonlinear solution, (--) linear solution.

The maximum dynamic displacement variation $(w)$ of mid-point of an inclined self-weight Timoshenko beam with inclination angle of $\theta=36 .^{\circ}$ vs. equivalent moving concentrated forces using both linear and nonlinear approaches for different velocity ratios of $\alpha=0.25,0.5,0.75,1$ and 1.25, are depicted in Fig. 6-a to 6-e, respectively. It can 


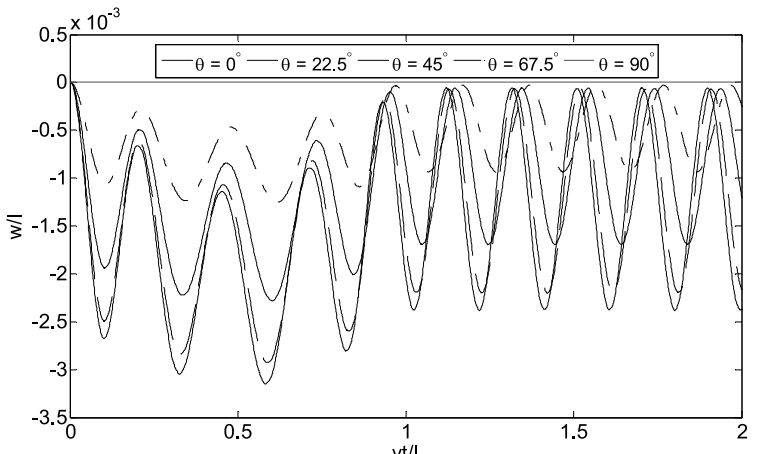

(a)

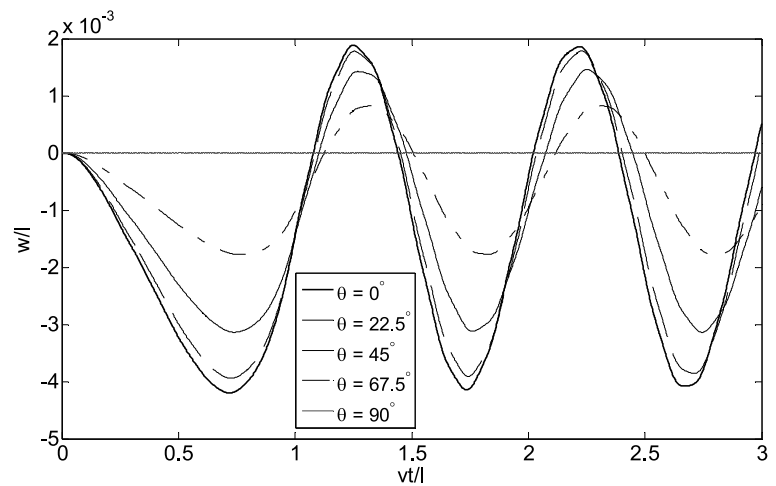

(c)

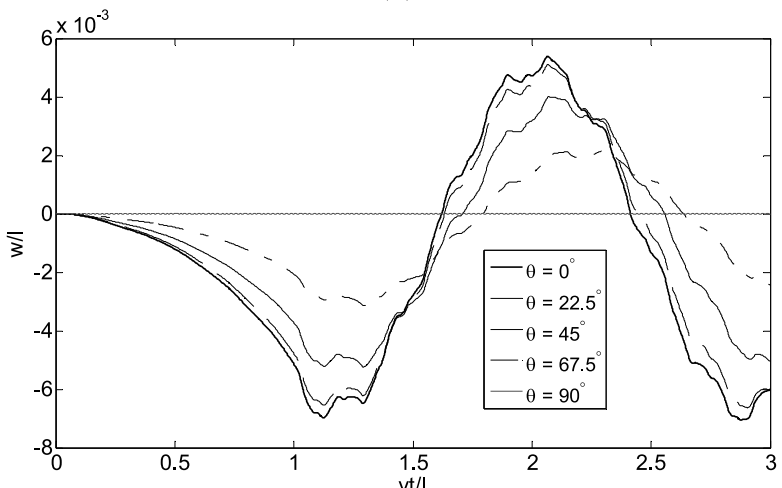

(e)

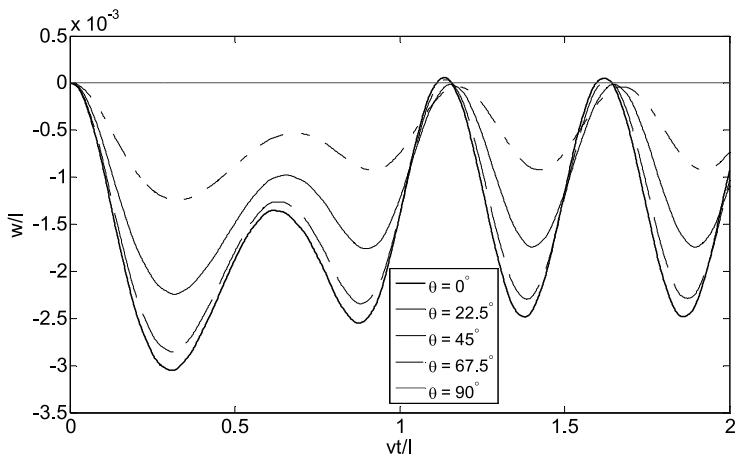

(b)

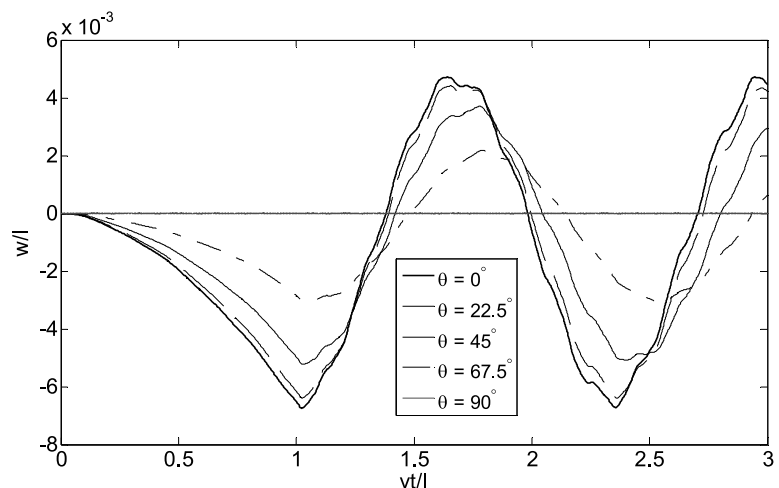

(d)

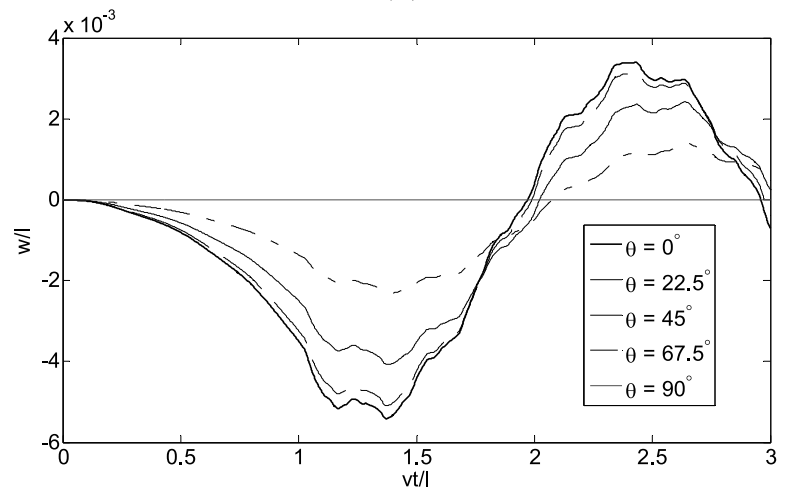

(f)

Fig. 7. Time histories for normalized mid-span deflection for $m=0.5 \rho A l$ (kg). (a) $\alpha=0.1$, (b) $\alpha=0.25$, (c) $\alpha=0.5$, (d) $\alpha=0.75$, (e) $\alpha=$ 1 , (f) $\alpha=1.25$. (一) $\theta=0^{\circ},(--) \theta=22.5^{\circ},(--) \theta=45^{\circ},(--) \theta=67.5^{\circ},(-) \theta=90^{\circ}$.

be seen that the dynamic deflection of the nonlinear analysis is always lower than the one obtained from the linear solution. As it was stated for Fig. 5, this incident which is known as the hardening behavior is mostly due to the existence of the coupled quadratic-cubic nonlinearity characteristic in the equations of motion of the beam. Also, it is seen from this figure that the dynamic mid-point displacements of such beam using linear and nonlinear solutions are almost the same for the value of $F \leqslant 0.3 \rho g A l$. However, after this point, the magnitude of the $w$ of the nonlinear and the linear solutions differs gradually and the difference grows rapidly as the value of $F$ increases. Also, it can be observed from Fig. 6 that the difference between the linear and the nonlinear solutions has an increasing trend up to the load velocity ratio of $\alpha=0.5$ and a reverse trend afterwards. It should be noted that the maximum difference between linear and nonlinear solutions for all cases in this figure occurs at $F=2.5 \rho g A l$ at $\alpha=0.5$ (see Fig. 6-b). 
Table 2

$\delta$ variation in targeting of planar projectile on an inclined Timoshenko beam for various $\alpha$ using nonlinear analysis for moving mass model; $\left(v_{c r}=131.60 \mathrm{~m} / \mathrm{s}\right)$

\begin{tabular}{|c|c|c|c|c|c|c|}
\hline \multirow{3}{*}{$\begin{array}{l}\text { Velocity } \\
\text { ratio } \alpha\end{array}$} & \multicolumn{6}{|c|}{$\delta(+$ or -$)(\mathrm{m})$ due to moving mass of $m=2 \rho A l(\mathrm{~kg})$} \\
\hline & \multicolumn{2}{|c|}{$\theta=36^{\circ}$} & \multicolumn{2}{|c|}{$\theta=45^{\circ}$} & \multicolumn{2}{|c|}{$\theta=54^{\circ}$} \\
\hline & $\begin{array}{c}\text { weightless } \\
\text { beam }\end{array}$ & $\begin{array}{l}\text { self-weight } \\
\text { beam }\end{array}$ & $\begin{array}{l}\text { weightless } \\
\text { beam }\end{array}$ & $\begin{array}{c}\text { self-weight } \\
\text { beam }\end{array}$ & $\begin{array}{c}\text { weightless } \\
\text { beam }\end{array}$ & $\begin{array}{c}\text { self-weight } \\
\text { beam }\end{array}$ \\
\hline 2 & 96.59 & 127.83 & -6.16 & -11.45 & -79.82 & -110.78 \\
\hline 2.25 & 107.82 & 145.46 & -5.94 & -11.42 & -87.64 & -123.55 \\
\hline 2.5 & 115.98 & 160.24 & -5.47 & -10.97 & -92.85 & -133.59 \\
\hline 2.75 & 121.97 & 172.47 & -4.92 & -10.29 & -96.31 & -141.41 \\
\hline 3 & 125.38 & 182.76 & -4.30 & -9.54 & -97.83 & -147.72 \\
\hline
\end{tabular}

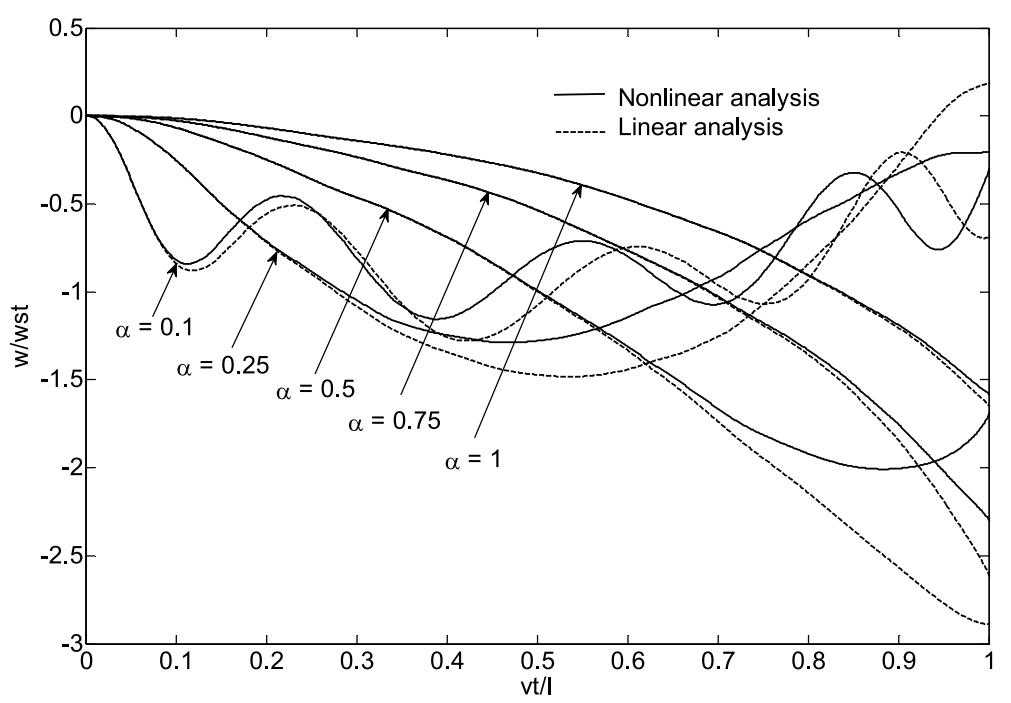

Fig. 8. Time histories for normalized mid-point deflection of an inclined self-weight Timoshenko beam, $\theta=36^{\circ}, m=1.2 \rho A l(\mathrm{~kg})$. ( $\longrightarrow$ ) nonlinear analysis, $(-)$ linear analysis.

Moreover, as it can be seen that the variation of the linear solution mathematically does not follow a linear trend in Figs 5 and 6, whereas this variation does not represent in a weightless Timoshenko beam.

Figure 7 (a-f) show the time histories for normalized dynamic deflection $(w / l)$ of the mid-point of the beam vs. non-dimensional time $v t / l$, for different values of beam's inclination angle $\left(\theta=0^{\circ}, 22.5^{\circ}, 45^{\circ}, 67.5^{\circ}\right.$ and $\left.90^{\circ}\right)$ and velocity ratios ( $\alpha=0.1,0.25,0.5,0.75,1$ and 1.25$)$ of the traveling mass value of $m=0.5 \rho A l$ using nonlinear analysis. It should be mentioned that $t$ denotes the time from the moment when the moving mass enters the beam. By an overall inspection of Figs 7-a to 7-f in the region of $0 \leqslant v t / l \leqslant 1$ one can say that by increasing the beam's inclination angle the dynamic displacement of beam's mid-point decreases and for example at $\theta=90^{\circ}$, the dynamic lateral displacement becomes zero as expected regardless of time marching. Furthermore, it can be seen that at each velocity ratio, the peak point of all curves does not occur at the same $v t / l$, no matter what the beam inclination angle would be. Also it can be seen that for $\alpha>0.5$ while the mass leaves the beam $(v t / l>1)$, the maximum dynamic displacement of mid-point of the beam become larger than those obtained while $v t / l<1$, no matter what the beam inclination angle would be.

Figure 8 illustrates the variation of $w / w_{s t}$ of the mid-point of an inclined beam with inclination angle of $\theta=36^{\circ}$ vs. vtll for different velocity ratios of the traveling mass value of $m=1.2 \rho \mathrm{Al}$. From this figure, one can conclude that the results for the lateral dynamic displacement obtained by the nonlinear analysis almost represent smaller values than those calculated by linear analysis. Also, it can be seen that the peak value of each curve does not occur at the same vtll. Moreover, variation of the values of these peak points have an increasing trend up to the load velocity ratio of $\alpha=0.5$. In addition, it is seen that for higher velocity ratio, i.e. $\alpha=1$, the lateral dynamic displacement of the beam's mid-point tends to a very small value at the time of leaving the beam which means that the beam does 


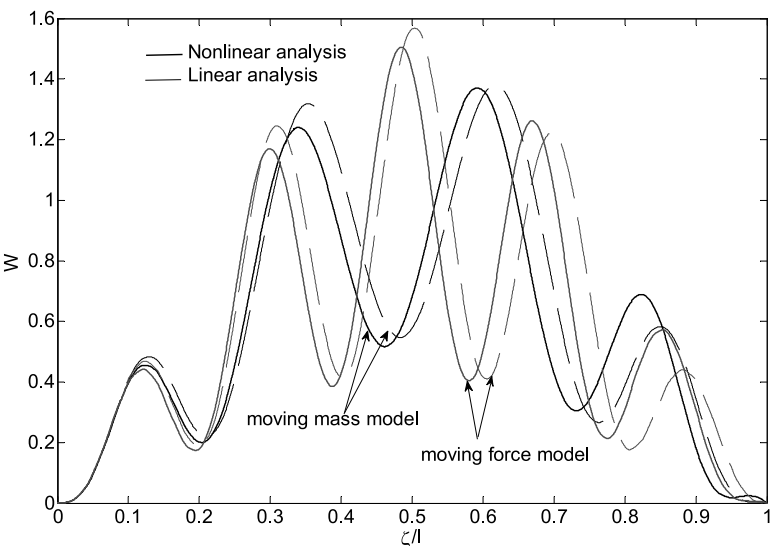

(a)

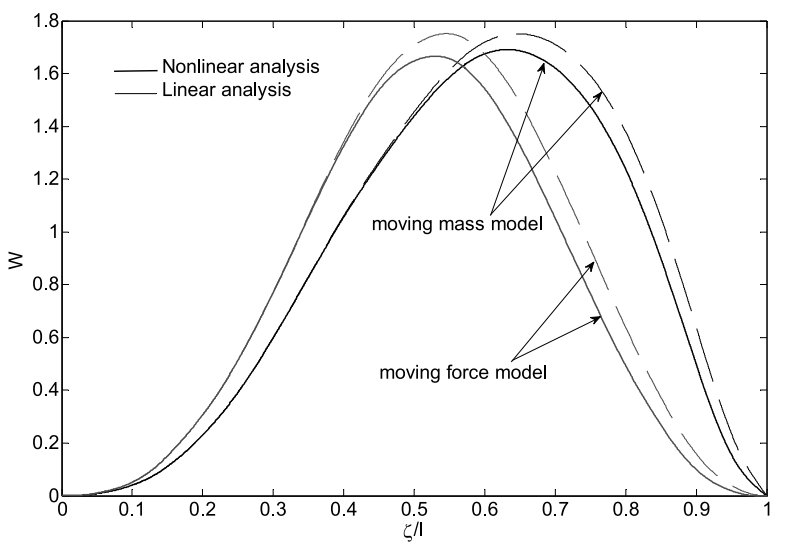

(c)

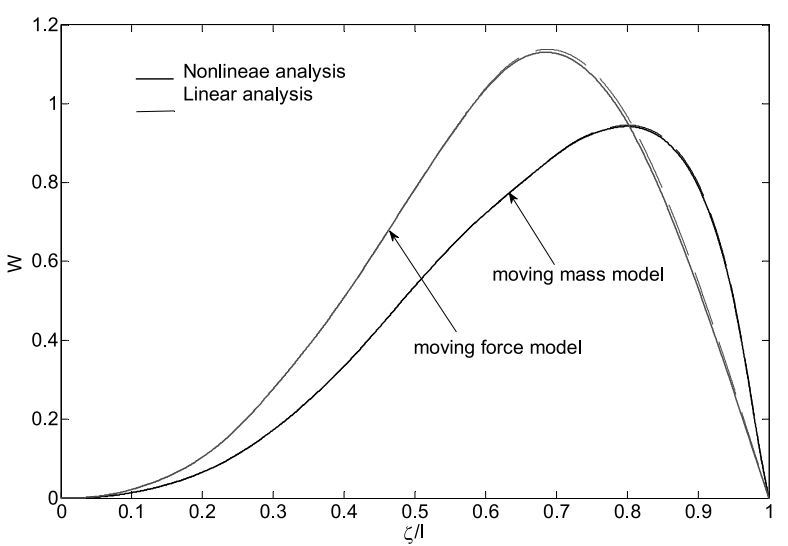

(e)

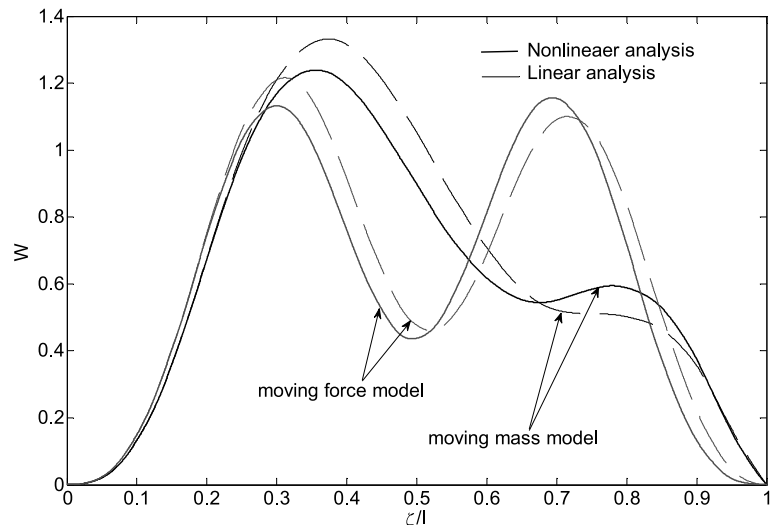

(b)

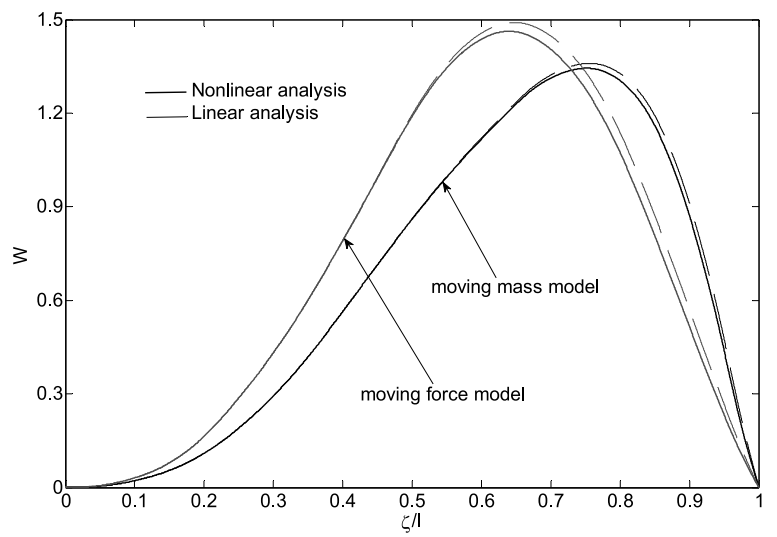

(d)

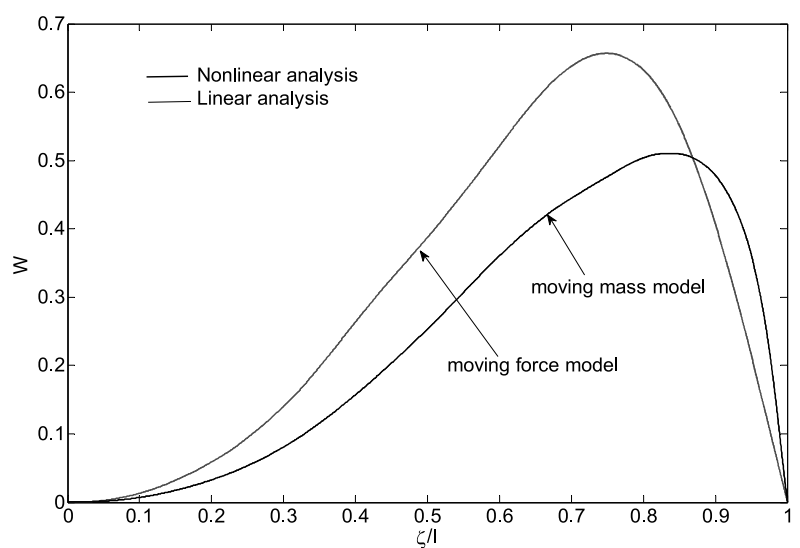

(f)

Fig. 9. Normalized vertical deflection $\left(W=w_{d} / w_{s t}\right)$ vs. $\zeta(t) / l$ under a moving load, $\theta=36^{\circ}$; (a) $\alpha=0.1$, (b) $\alpha=0.25$, (c) $\alpha=0.5$, (d) $\alpha=$ 0.75 , (e) $\alpha=1$, (f) $\alpha=1.5$. (-) nonlinear analysis, (----) linear analysis.

not have enough time to respond accordingly against the fast speed of the moving mass. Finally, according to the nonlinear analysis the maximum downward beam's mid-point dynamic displacement occurs while the mass leaves the beam with the velocity ratio of $\alpha=0.75$.

Figure 9 shows the variation of normalized lateral dynamic displacement $\left(W=w_{d} / w_{s t}\right)$ of an inclined beam 
Table 3

$\delta$ variation in targeting of planar projectile on an inclined Timoshenko beam for various $\alpha$ using nonlinear analysis for moving force model; $\left(v_{c r}=131.60 \mathrm{~m} / \mathrm{s}\right)$

\begin{tabular}{|c|c|c|c|c|c|c|}
\hline \multirow{3}{*}{$\begin{array}{l}\text { Velocity } \\
\text { ratio } \alpha\end{array}$} & \multicolumn{6}{|c|}{$\delta(+$ or -$)(\mathrm{m})$ due to moving force of $F=2 \rho \mathrm{g} A l(\mathrm{~N})$} \\
\hline & \multicolumn{2}{|c|}{$\theta=36^{\circ}$} & \multicolumn{2}{|c|}{$\theta=45^{\circ}$} & \multicolumn{2}{|c|}{$\theta=54^{\circ}$} \\
\hline & $\begin{array}{l}\text { weightless } \\
\text { beam }\end{array}$ & $\begin{array}{l}\text { self-weight } \\
\text { beam }\end{array}$ & $\begin{array}{l}\text { weightless } \\
\text { beam }\end{array}$ & $\begin{array}{l}\text { self-weight } \\
\text { beam }\end{array}$ & $\begin{array}{l}\text { weightless } \\
\text { beam }\end{array}$ & $\begin{array}{c}\text { self-weight } \\
\text { beam }\end{array}$ \\
\hline 2 & 15.26 & 20.05 & -0.13 & -0.23 & -11.29 & -14.94 \\
\hline 2.25 & 17.92 & 22.91 & -0.15 & -0.24 & -13.25 & -17.02 \\
\hline 2.5 & 20.14 & 25.43 & -0.15 & -0.24 & -14.86 & -18.18 \\
\hline 2.75 & 20.43 & 25.96 & -0.12 & -0.21 & -15.04 & -19.18 \\
\hline 3 & 22.10 & 27.78 & -0.12 & -0.20 & -16.25 & -20.49 \\
\hline
\end{tabular}

with $\theta=36^{\circ}$ under the moving mass of $m=0.5 \rho \mathrm{Al}$ and as well as an equivalent concentrated moving force of $F=0.5 \rho g A l$ vs. normalized instantaneous mass/force position i.e, $x=\zeta(t) / l$, using linear and nonlinear analysis. The lateral static displacement at $x=l / 2$ for a simply supported Euler-Bernoulli beam is given by: $w_{s t}=$ $m g l^{3} \cos \theta / 48 E I+5 \rho g A l^{4} \cos \theta / 384 E I$. From this figure it can be seen that there are significant discrepancies between the deflection under the moving mass and the deflection under the equivalent concentrated moving force, especially towards the later part of the motion. Also as it can be observed from Fig. 9 that the maximum lateral dynamic displacement of the beam has an increasing trend up to the velocity ratio of $\alpha=0.5$ and a reverse trend afterwards. Moreover, the deflection under the equivalent concentrated moving force is not an upper bound for the deflection under the moving mass as reported by other researchers [9].

\section{Case study: accuracy of targeting of repetitive projectiles leaving an inclined beam}

The general application of this study can be addressed to the dynamic analysis of any kind of low size to high size caliber ammunition used in different types of fire arms weaponry. Consider a planar motion of a simple projectile with the mass of $m$ having a known and constant velocity $v$ traveling on an inclined beam as shown in Fig. 10. Usually in a situation like this the accuracy of the expected impact point is the most important objective for this kind of motion. Since we are not going to consider the beam as a rigid continuum therefore, it is clear that the beam deflection and its slope at the end supports have to be considered when the projectile leaves the beam. We intend to show the effect of this slope on the target accuracy using nonlinear analysis. Suppose that point $p_{1}$ is the position of the impact point predicted by the rigid type of analysis and point $p_{2}$ the same point predicted by the nonlinear analysis under beam's inclination angle of $\theta_{\text {rigid }}$ and beam's end slope of $\theta_{\text {endslope }}$ (see Fig. 10) [25].

Table 2 shows the calculated results for the difference in target position $\delta\left(\delta=R_{\text {nonlinear }}-R_{\text {rigid }}\right)$ vs. the velocity ratios $\alpha$ for a Timoshenko beam under the act of a moving mass with different inclination angles of $\theta_{\text {rigid }}=$ $36^{\circ}, 45^{\circ}$ and $54^{\circ}$, respectively. The following data is used for this case study: $I=606 \times 10^{-8} \mathrm{~m}^{4}, A=25.3 \times$ $10^{-4} \mathrm{~m}^{2}, l=6 \mathrm{~m}, m=2 \rho A l \mathrm{~kg}$ and $k=0.2093$. To illustrate the effect of considering the slope of the beam at the time when the projectile leaves the tip of the beam, consider an instance where $\alpha=3$ and $\theta_{\text {rigid }}=36^{\circ}$, and keeping all other parameters the same, the difference in target position with respect to the rigid beam assumption considering both weightless beam and the self-weight beam models are $125.38 \mathrm{~m}$ and $182.76 \mathrm{~m}$, respectively which have about $0.83 \%$ error and $1.21 \%$ error, respectively (see Fig. 10). As can be seen from Table 2, under considered range for the velocity ratios the maximum absolute values of $\delta$ occur at $\alpha=3$ at $\theta=36^{\circ}$. Moreover, this table further reveals that for three different inclination angles the $\delta$ has its absolute least value at $\theta=45^{\circ}$ at any velocity ratios. It can also be observed from this table that for each beam's inclination angle the size of $\delta$ obtained by self-weight beam model is always greater than the size of $\delta$ obtained by weightless beam model. The reason for this added difference is due to the further created end slope of the beam using self-weight beam model when the moving mass leaves the platform.

Table 3 shows the calculated results for the difference in target position $\delta$ vs. the velocity ratios $\alpha$ for a Timoshenko beam under the act of a moving force with different inclination angles of $\theta_{\text {rigid }}=36^{\circ}, 45^{\circ}$ and $54^{\circ}$, respectively with all other parameters are the same. Moreover, by comparison of Tables 2 and 3 it can be seen that at each beam inclination angle the size of $\delta$ using moving force model is always smaller than those obtained from the moving mass model. 


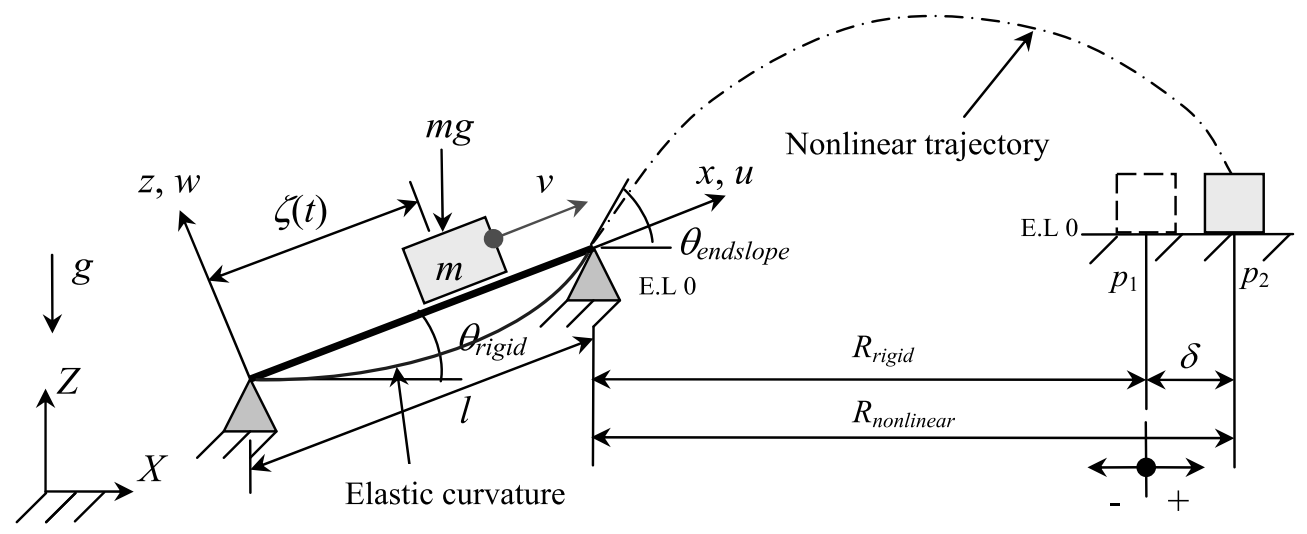

Fig. 10. Prediction of a planar motion of a simple projectile using different theories.

\section{Conclusions}

Three nonlinear coupled partial differential equations of motions for the rotation of warped cross-section, longitudinal and transversal displacements of an inclined pinned-pinned self-weight Timoshenko beam subjected to a moving mass as well as equivalent concentrated force were solved and the outcome results are as the followings:

1. It can be seen that by increasing the beam's inclination angle the dynamic lateral displacement of mid-point of the beam decreases and for example at $\theta=90^{\circ}$, it becomes zero as expected regardless of time marching (outcome of Fig. 7).

2. It can be observed that at any mass velocity, the dynamic magnification factor $D_{d}$ of the linear and nonlinear solutions have the lowest difference for small values of the moving mass $(m=0.25 \rho A l)$. However, for larger values of the moving mass $(m>0.25 \rho \mathrm{Al})$ the size of $D_{d}$ using nonlinear theory has lower values than those given by linear theory, hence the difference between the $D_{d}$ responses of the nonlinear and linear theories increase vividly (outcome of Fig. 3).

3. It can be seen that the dynamic mid-point displacements of a moving mass problem and a moving force problem using linear and nonlinear solutions have the lowest difference for the value of $m=0.2 \rho \mathrm{Al}$ and $F=0.3 \rho \mathrm{gAl}$, respectively. However, for the value of $m>0.2 \rho A l$ and $F>0.3 \rho g A l$, the magnitude of the beam deflection of the nonlinear and the linear solutions differs gradually and the difference grows rapidly as the value of $m$ and $F$ increases, respectively. Moreover, it can be seen that the variation of the linear solution mathematically does not follow a linear trend in a moving mass/force problem for an inclined self-weight beam (outcome of Figs 5 and 6).

4. Due to the existence of the quadratic-cubic nonlinearity nature of the governing coupled PDEs of motion, the system behaves like a hard spring. That is by increasing the magnitude of the moving mass or an equivalent concentrated moving force, the dynamic deflections become smaller than those from solution of linear system.

5. It can be seen that there are significant discrepancies between the instantaneous deflection under the moving mass and the equivalent concentrated moving force, especially towards the later part of the motion. Moreover, the deflection under the equivalent concentrated moving force is not an upper bound for the deflection under the moving mass (outcome of Fig. 9).

6. It has been noticed that by neglecting the nonlinear nature of the motion in an inclined Timoshenko beam, the weight of the beam and the effect of the end slope, one would get some error in the final position of a projectile at the impact point.

\section{References}

[1] L. Fryba, Vibration of Solids and Structures under Moving Loads, Thomas Telford Publishing, London, 1999. 
[2] M.H. Kargarnovin and D. Younesian, Dynamics of Timoshenko beams on Pasternak foundation under moving load, Mechanics research communications 31 (2004), 713-723.

[3] M.A. Foda and Z. Abduljabbar, A dynamic green function formulation for the response of a beam structure to a moving mass, Journal of Sound and Vibration 210 (3) (1998), 295-306.

[4] G. Michaltsos, D. Sophianopoulos and A.N. Kounadis, The effect of a moving mass and other parameters on the dynamic response of a simply supported beam, Journal of Sound and Vibration 191 (3) (1996), 357-362.

[5] A. Kidarsa, M.H. Scott and H.C. Christopher, Analysis of moving loads using force-based finite elements, Finite Elements in Analysis and Design 44 (4) (2008), 214-224.

[6] S.H. Ju, H.T. Lin, H.H. Hsueh and S.H. Wang, A simple finite element model for vibration analyses induced by moving vehicles, International for Numerical Methods in Engineering 68 (2006), 1232-1256.

[7] Y.H. Lin and M.W. Trethewey, Finite element analysis of elastic beams subjected to moving dynamic loads, Journal of Sound and Vibration 136 (2) (1990), 323-242.

[8] D. Thambiratnam and Y. Zhuge, Dynamic analysis of beams on an elastic foundation subjected to moving loads, Journal of Sound and Vibration 198 (2) (1996), 149-169.

[9] H.P. Lee, The dynamic response of a Timoshenko beam subjected to a moving mass, Journal of Sound and Vibration 198 (2) (1996), 249-256.

[10] P. Lou, G.L. Dai and Q.Y. Zeng, Finite-element analysis for a Timoshenko beam subjected to a moving mass, Journal of Mechanical Engineering Science, IMechE 220 (C) (2006), 669-678.

[11] R.T. Wang and T.H. Chou, Non-linear vibration of Timoshenko beam due to moving force and the weight of beam, Journal of Sound and Vibration 218 (1) (1998), 117-131.

[12] E. Esmailzadeh and M. Ghorashi, Vibration analysis of a Timoshenko beam subjected to a traveling mass, Journal of Sound and Vibration 199 (4) (1997), 615-628.

[13] E. Esmailzadeh and M. Ghorashi, Vibration analysis of beams traversed by uniform partially distributed moving masses, Journal of Sound and Vibration 184 (1) (1995), 9-17.

[14] R.T. Wang, Vibration of multi-span Timoshenko beams to a moving force, Journal of Sound and Vibration 207 (5) (1997), $731-742$.

[15] R.T. Wang and J.S. Lin, Vibration of multi-span Timoshenko frames due to moving loads, Journal of Sound and Vibration 212 (5) (1998), 417-434.

[16] X. Xu, W. Xu and J. Genin, A non-linear moving mass problem, Journal of Sound and Vibration 204 (3) (1997), $495-504$.

[17] M. Olsson, On the fundamental moving load problem, Journal of Sound and Vibration 145 (2) (1991), $299-307$.

[18] M.H. Kargarnovin, D. Younesian, D.J. Thompson and C.J.C. Jones, Response of beams on nonlinear viscoelastic foundations to harmonic moving loads, Computers \& Structures 83 (2005), 1865-1877.

[19] D. Younesian, M.H. Kargarnovin, D.J. Thompson and C.J.C. Jones, Parametrically excited vibration of a Timoshenko beam on random viscoelastic foundation subjected to a harmonic moving load, Nonlinear Dynamics 45 (2006), 75-93.

[20] J.J. Wu, Dynamic analysis of an inclined beam due to moving loads, Journal of Sound and Vibration 288 (2005), 107-133.

[21] A.N. Yanmeni Wayou, R. Tchoukuegno and P. Woafo, Non-linear dynamics of an elastic beam under moving loads, Journal of Sound and Vibration 273 (2004), 1101-1108.

[22] M. Simsek and T. Kocaturk, Nonlinear dynamic analysis of an eccentrically prestressed damped beam under a concentrated moving harmonic load, Journal of Sound and Vibration 320 (2009), 235-53.

[23] J.W. Hijmissen and W.T. van Horssen, On transverse vibrations of a Timoshenko beam, Journal of Sound and Vibration 314 (2008), 161-179.

[24] K. Kiani, A. Nikkhoo and B. Mehri, Prediction capabilities of classical and shear deformable beam models excited by a moving mass, Journal of Sound and Vibration 320 (2009), 632-648.

[25] A. Mamandi, M.H. Kargarnovin, D. Younesian, Nonlinear dynamics of an inclined beam subjected to a moving load, Nonlinear Dynamics 60 (2010), 277-293.

[26] A. Ansari, E. Esmailzadeh and D. Younesian, Internal-external resonance of beams on non-linear viscoelastic foundation traversed by moving load, Nonlinear Dynamics 61 (2010), 163-182.

[27] A.H. Nayfeh and D.T. Mook, Nonlinear Oscillations, Wiley-Interscience, New York, 1995.

[28] L. Meirovitch, Principles and Techniques of Vibrations, Prentice-Hall. Inc., New Jersey, 1997.

[29] A.O. Cifuentes, Dynamic response of a beam excited by a moving mass, Finite Element in Analysis and Design 5 (1989), $237-246$. 

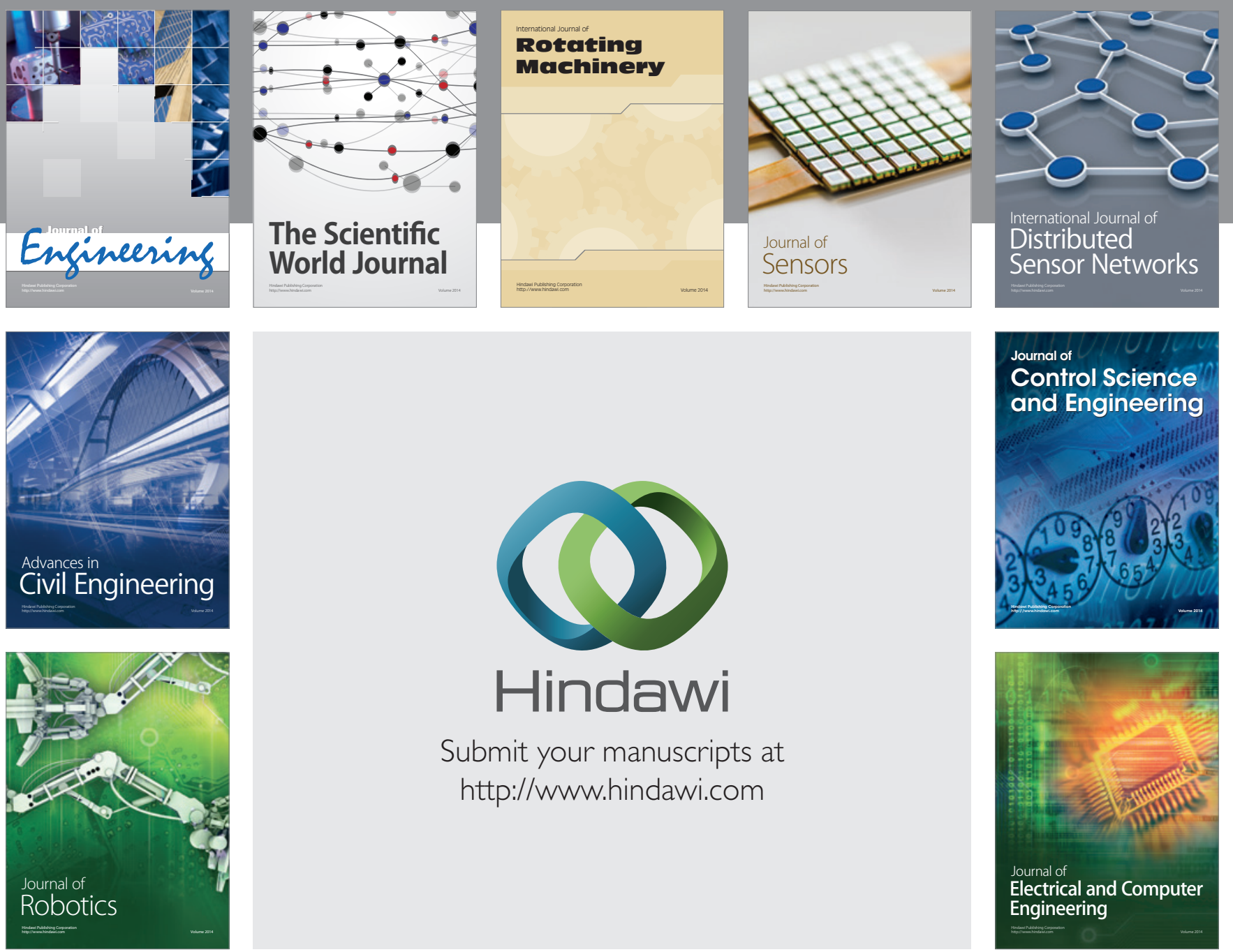

Submit your manuscripts at

http://www.hindawi.com
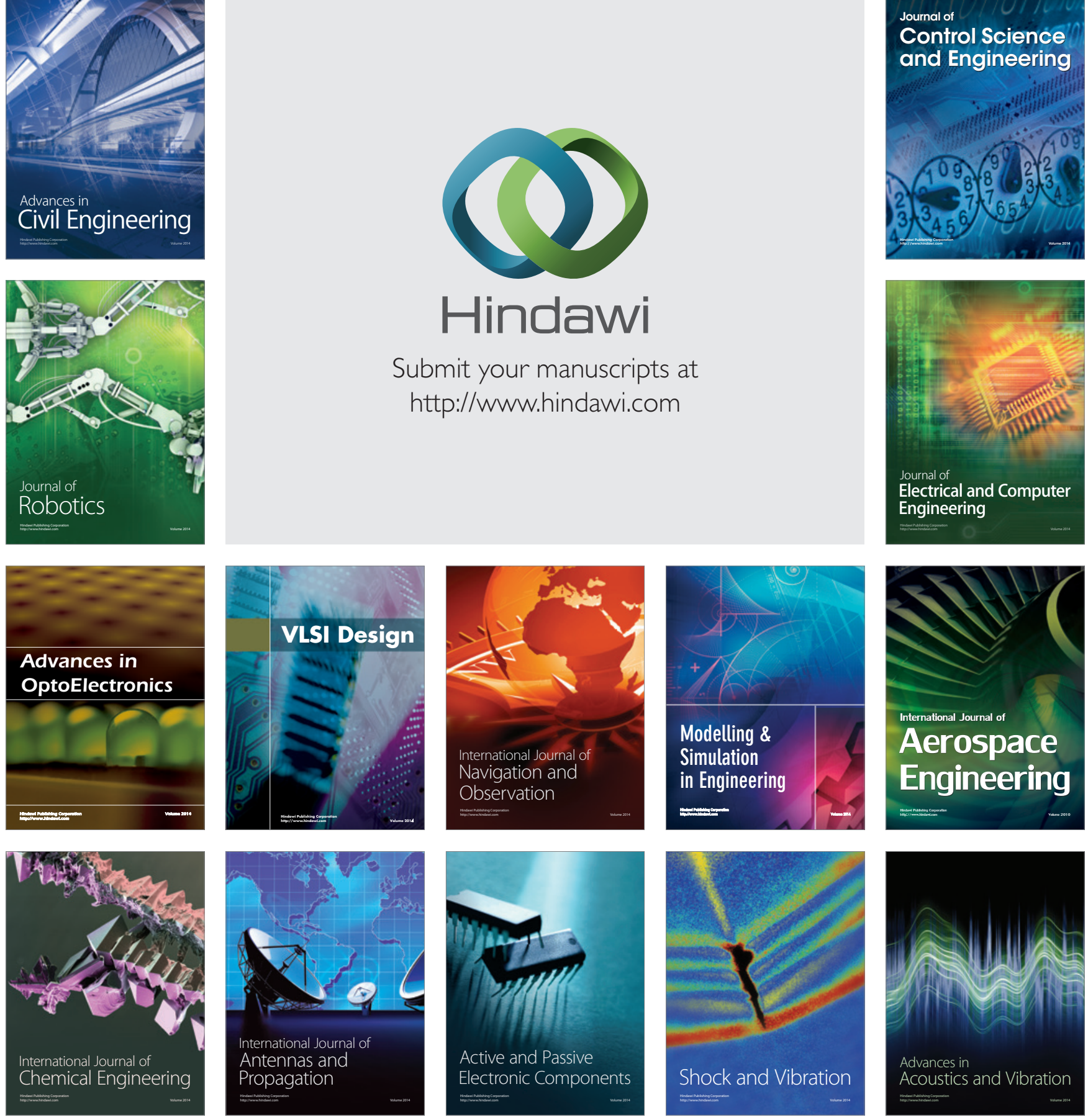